\title{
Dynamics in a disease transmission model coupled virus infection in host with incubation delay and environmental effects
}

\author{
Abulajiang Aili ${ }^{1} \cdot$ Zhidong Teng $^{1} \cdot$ Long Zhang $^{1}$ \\ Received: 7 November 2021 / Revised: 28 December 2021 / Accepted: 24 January 2022 / \\ Published online: 9 February 2022 \\ (c) The Author(s) under exclusive licence to Korean Society for Informatics and Computational Applied \\ Mathematics 2022
}

\begin{abstract}
In this paper, a disease transmission model coupled virus infection in host with incubation delay and environmental effects is studied. For the virus infection model in host with immune, latent delay and environmental virus invading, the threshold criteria on the global stability of antibody-free and antibody response infection equilibria are established. For the disease transmission model with incubation delay and immune response in host, basic reproduction number $R_{0}$ is defined, and the local stability of equilibria are established, i.e., the disease-free equilibrium is locally asymptotically stable if $R_{0}<1$, and the endemic equilibrium is locally asymptotically stable if $R_{0}>1$. Furthermore, the uniform persistence of positive solutions is studied while there is not the direct transmission of disease by the infected individuals. Finally, the numerical examples are presented to verify the main results.
\end{abstract}

Keywords Disease transmission model · Virus infection model · Immunity response $\cdot$ Incubation delay · Environmental effects $\cdot$ Stability · Uniform persistence

MSC Classification 34D20 $\cdot$ 34D23 $\cdot$ 92B05 $\cdot 92 \mathrm{D} 30$

\section{Introduction}

As is well-known, infectious diseases have a great impact on humans health, social development and national security. Especially, over the past few decades, human beings have been faced with serious threat from various kind of infectious diseases which are primarily caused through the virus, such as AIDS, Ebola, SARS, MERS and COVID-

Long Zhang

longzhang_xj@sohu.com

1 College of Mathematics and System Science, Xinjiang University, Shui Mo Gou Street, Urumqi 830046, Xinjiang, People's Republic of China 
19, etc (See [1-5]). In order to effectively control the prevalence of infectious diseases, it is necessary to conduct in-depth research on the characteristics, transmission rules and treatment measures of infectious diseases. Using mathematical models as a tool to study various problems in infectious diseases can make people have more comprehensive and profound understanding of the occurrence and spread of infectious disease from quantitative perspective. The epidemic dynamical models in the form of differential equations and difference equations are an important method for quantitative research on infectious diseases. The function of this method not only provide theoretical and practical basis for control the outbreak of disease, but also help to explore the effects of infectious disease prevention and control measures in various aspects, and enrich the understanding of prevention and control decisions.

Many infectious diseases usually involves at least two key processes, such as Hepatitis, Ebola, COVID-19, etc. One is the within-host virus infection process in which the virus growth, spreads or be eliminated in the infected host, where the host's immunity plays a vital role in the growth, spread or elimination of virus. The other one is the between-host disease transmission process in which the susceptible individual is infected through close human contact or environmental virus. Rhoubari et al. [6] have proposed two mathematical Ebola virus disease (EVD) model with three modes of transmission to explain that the susceptible is infected by Ebola by contact with bodily fluids of infected individual, infectious corpse or contaminated environment. In [7-9], the authors pointed out that growth and spread of virus in host is a rapid process, while the spread of diseases between-host is a relatively slow process.

In $[7,10]$, the authors proposed the following mathematical model coupling withinand between-host dynamics including a disease-induced death rate for the hosts, it extends the model which was studied in $[11,12]$. This model can be coupled with environmental virus as is given below

$$
\left\{\begin{array} { r l } 
{ \frac { d T } { d s } } & { = \Lambda _ { c } - k V T - m T , } \\
{ \frac { d T ^ { * } } { d s } } & { = k V T - ( m + d ) T ^ { * } , } \\
{ \frac { d V } { d s } } & { = g ( E ) + p T ^ { * } - c V , }
\end{array} \quad \left\{\begin{array}{l}
\frac{d S}{d t}=\Lambda-\lambda E S-\mu S, \\
\frac{d I}{d t}=\lambda E S-(\mu+\alpha) I, \\
\frac{d E}{d t}=\theta I V(1-E)-\gamma E,
\end{array}\right.\right.
$$

where $T=T(s), T^{*}=T^{*}(s)$ and $V=V(s)$ represent the densities of healthy cells, infected cells and viral load at time $s, S(t)$ and $I(t)$ represent the numbers of susceptible and infected hosts at time $t$, and $E(t)$ denotes the level of environment contamination at time $t$. The parameter $\Lambda_{c}$ is the recruitment of $T(s), k$ is the infection rate of cells, $m$ is the natural mortality of cells, $d$ is the infection-induced mortality rate of cells, $p$ is parasite production rate by an infected cell, $c$ is the within-host parasite clearance rate, $\lambda$ is infection rate of individuals at population level, $\Lambda$ is the recruitment of hosts, $\mu$ is the natural death rate of hosts, $\alpha$ is the disease-induced death rate of hosts, and $\gamma$ is clearance rate of the environment. The function $g(E)$ is an added rate in the change of parasite load due to the continuous ingestion of parasites by the host from a contaminated environment. By using the limit equation theory and singular perturbation equation theory of differential equations (See [13- 
18]), the authors firstly decomposed the whole model into fast time sub-model and slow time sub-model, and further studied the both sub-models and whole coupling model, respectively. The existence, local and global stability of equilibria, backward bifurcation and virulence evolution of sub-models and the whole coupled model are emphasized.

Recently, the epidemic models with two processes of within-host virus infection and between-host disease transmission have been investigated in many articles (See [7-12, 19-24]). Particularly, Wen et al. [19] investigated a discrete time environmentallydriven coupling dynamic model of within-host virus infection and between-host disease transmission. Wen et al. [20] further improved the above discrete time model into a more practical form with saturation incidence. In $[8,9]$, the authors also investigated the age-structured and reaction-diffusion epidemic models for coupling withinand between-host dynamics in environmentally-driven infectious diseases.

In many infectious diseases, the incubation period can not be neglected, such as Influenza, COVID-19, etc., these diseases have no obvious symptoms in the early stages of infection, some symptoms only appear after a period of incubation, and some of them are not only contagious during its infectious period, but also during its incubation period. Particularly, such as COVID-19 is contagious during its incubation period [25]. And with the idea of infectivity in incubation period, Jiao et al. [26] proposed a SEIR epidemic model with homestead-isolation on the susceptible. However, for some diseases, such as Tuberculosis, Measles and AIDS, on adequate contact with an infective, a susceptible individual becomes lurks, that is, infected but not infective. This individual remains in incubation period before becoming infective (See [27, 28]), and this incubation period can be modeled by incorporating it as a delay effect. Xu et al. [29] concerned with the combined effects of saturation incidence rate and time delay representing the incubation period of the disease on the dynamics of an epidemiological model. Cooke et al. [30] proposed a SEIR epidemic model with time delays to investigate the influence of incubation period on the spread of infectious diseases.

We already knew, the immune response has a great role in controlling the spread of disease during the virus infection process (See [31-42]). Particularly, when the antibody in host does not work or the effect of the antibody is weak, then the virus will continue to spread. As a result, the spread of disease between-host will be difficult to control. Otherwise, when the host have an extensive antibody response, the production rate of B cells in host will be increased, and environmental virus invade into host will be prevented, then the transmission of disease is well controlled. Based on the fact that humoral immunity is much more efficient than cellular immunity in some infection [41], Murase et al. [42] introduced a model that described the interaction between target cell, pathogens and immune responses. Wang et al. [34] applied this model to investigate the dynamical behavior of in host virus infection models with humoral immunity and intracellular delays. Hattaf [40] proposed a generalized viral infection model with general incidence functions for both modes of transmission humoral immunity, two distributed intracellular delays and one discrete immunological delay.

In this paper, we will study more general disease transmission model coupled virus infection in host with incubation delay and environmental effects. This epidemic model 
is coupled with the virus infection model via the environmentally-driven infectious diseases. We will use the limit system method to investigate this coupled model, and see that the immune response for the dynamical behavior of between-host disease transmission come into the important influence for the extinction and persistence of disease.

The structure of this paper is as follows. In Sect. 2, we give a detailed description for the disease transmission model with incubation delay and environmental effects. In Sect. 3, we discuss the virus infection model in host with immune, latent delay and environmental virus invading, the threshold criteria on the global stability of antibody-free and antibody response infection equilibria are established. In Sect. 4, we study the disease transmission model with incubation delay and immune response, the nonnegative, boundness of solution, and the local stability of equilibria are proved. Beside, we prove the uniform persistence of positive solutions while there is not the direct transmission of disease by infected individual. In Sect. 5, the theoretical results are illustrated by numerical simulations. In Sect. 6, a brief conclusion is given.

\section{Model description}

In this part, based on the above discussion, we propose a disease transmission model coupled virus infection in host, which includes the latent delay and antibody immune in virus infection, the incubation delay and environmental effects in disease transmission. Firstly, we put forward the following assumptions.

1. The total population is divided into the susceptible, infected and removed. They have same natural mortality rate. The susceptible has a recruitment rate. The viruses are divided into the virus in human body and the virus in human life environment. They cause the virus infection in human and environmental pollution, respectively.

2. The transmission of disease is caused by susceptible exposed to virus in the environment and the direct infection from close contact with infected individual. The incidence rates of two transmission routes are bilinear.

3. After being infected by the virus, the susceptible become infected after an average incubation period. During this incubation period there is only natural death, and no disease transmission. The infected has disease-induced death and also can be cured as a removed by treatment and autoimmunity. We assume that the removed has lifelong immunity and will not return to the susceptible, and the disease will not relapse.

4. The environmental virus mainly comes from the emission of infected. The amount of effluent virus per unit time is proportional to the number of infected individual and the average virus load in infected individual. The environmental virus can be eliminated artificially and naturally.

5. The environmental virus can invade into the human body through breathing, eating, and contacting, etc., and making the number of virus in the human body gradually increase, and then causing the virus infection in host. 


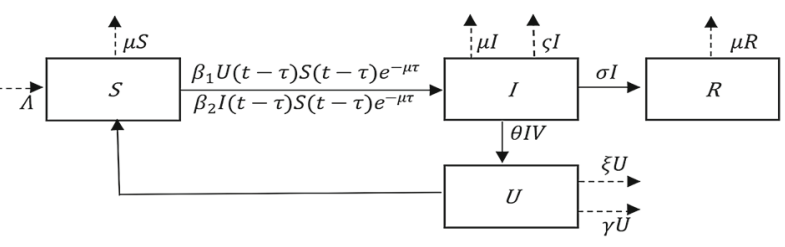

Fig. 1 The susceptible are produced at the rate of $\Lambda$, die at the rate of $\mu$ and become infected by environment contamination $U$ and close human contact at time $(t-\tau)$. In addition, $V$ indicates the total amount of virus in infected individual, $\theta$ indicates the emission rate of virus to the environment released by each infected individual, $\theta V I(t)$ indicates the increase of virus concentration in the environment per unit time as a whole

Based on above assumptions (1)-(4), we can construct the following warehouse diagram:

On account of above warehouse diagram, we can easily establish the following environmentally-driven disease transmission dynamical model with incubation delay, indirect and direct infection

$$
\left\{\begin{array}{l}
\frac{d S}{d t}=\Lambda-\beta_{1} U(t) S(t)-\beta_{2} I(t) S(t)-\mu S(t) \\
\frac{d I}{d t}=\left(\beta_{1} U(t-\tau) S(t-\tau)+\beta_{2} I(t-\tau) S(t-\tau)\right) e^{-\mu \tau}-(\mu+\sigma+\zeta) I(t) \\
\frac{d R}{d t}=\sigma I(t)-\mu R(t) \\
\frac{d U}{d t}=\theta I(t) V-(\xi+\gamma) U(t)
\end{array}\right.
$$

Where $S(t), I(t), R(t)$ and $U(t)$ represent the numbers of susceptible, infected, removed and the level of environment contamination at time $t$, respectively, $\beta_{1}$ is the infection rate of susceptible in contaminative environment, $\beta_{2}$ is the number of contacts by one infective per unit time multiplied by the probability that a contact with a susceptible leads to infection, $\tau$ is the incubation/latent period, $e^{-\mu \tau}$ is the surviving rate of infected hosts during incubation period $\tau, \Lambda$ is the recruitment rate of susceptible, $\mu$ is the natural mortality rate of total population, $\sigma$ is the quarantine and cure rate of infected individual, $\zeta$ is the disease-induced death rate of infected, $\xi$ is the decay rate of virus in the environment, $\gamma$ is the virus clearance rate in the environment. All these parameters are non-negative constants.

In model (2), there is a variable $V$ in dynamical equation of environmental virus $U$, which represents the total amount of virus in infected host. To get the exact change of $V$, we need to further study the dynamic process of virus infection in host. We have seen that dynamic models of virus infection in different types have been established in many articles (See [7-12, 19, 21, 22, 24]). In this paper, based on above assumptions (5), we propose the following within-host virus infection model with latent delay, 
antibody immune and environmental virus invading

$$
\left\{\begin{aligned}
\frac{d T}{d s} & =\Lambda_{c}-k V(s) T(s)-m T(s), \\
\frac{d T^{*}}{d s} & =k V\left(s-\tau_{1}\right) T\left(s-\tau_{1}\right) e^{-m \tau_{1}}-(m+d) T^{*}(s), \\
\frac{d V}{d s} & =g(U(t))+p T^{*}(s)-c V(s)-q B(s) V(s), \\
\frac{d B}{d s} & =\alpha B(s) V(s)-\omega B(s) .
\end{aligned}\right.
$$

Where we use letter $s$ to represent the dynamic evolution time of cells, virus, and $B$ cells in hosts, $T=T(s), T^{*}=T^{*}(s), V=V(s)$ and $B=B(s)$ represent the densities of healthy cells, infected cells, virus load and $B$ cells in host at time $s$, respectively, $q$ is the neutralization rate of $B$ cells, $\alpha$ is the production rate of $B$ cells, $\omega$ is mortality rate of $B$ cells. The other parameters $\Lambda_{c}, k, m, d, p$ and $c$ in model(3) are defined the same as those of model (1). All these parameters are non-negative constants. The function $g(U)$ in model (3) represents the increase in the number of virus in host caused by environmental virus invading into the host per unit time. The virus invasion usually indicates that the virus invading into host through breathing, eating, and contacting with air, water, food or other objects which contaminated by environmental virus. Obviously, as the number of environmental virus increases, the number of virus that invade into host will also increase. Therefore, we can make the following assumptions for function $g(U)$ :

$\left(\mathbf{H}_{1}\right) g^{\prime}(U)>0$ for all $U \geq 0$, and $g(0)=0$.

The simplest form of $g(U)$ studied in [10-12] is the linear function $g(U)=a U(a>$ 0 ), which means that the amount of environmental virus entering the host is linearly increasing with $U$. In addition to this, the other forms of $g(U)$ studied in [12] include $g_{1}(U)=\frac{a U}{1+b U}$ with constants $a>0$ and $b>0$, which means that the amount of environmental virus entering the host will reach a saturation level.

It is easy to see that there are some infectious diseases infected by virus, such as COVID-19, Hepatitis B, Zika, Ebola, etc. The process of virus infection in hosts needs to be described by virus infection model (3) with environmental virus effects. Particularly, in above diseases, people get infected from the virus through breathing, eating, and contacting with air, water, food or other objects which were contaminated by environmental virus $U$. Before isolation and treatment, the environmental virus $U$ will continuously invade into the host and gradually increase the amount of virus in host, where, the amount of virus increased per unit time can be represented by $g(U)$, and when the level of virus in the host reaches a certain level, the host will release the virus into the surrounding environment, and the environment eventually becomes contaminated with virus. 


\section{Virus infection model in host with immune, latent delay and environmental virus invading}

\subsection{Basic properties}

For virus infection model (3) with latent delay, immune and environmental virus invading, since the changes process of virus infection in host is much faster than the disease transmission process, we can assume that the environmental contamination rate $U(t)$ in model (3) remains a constant $U$. Thus, model (3) becomes into an isolated virus infection model in host.

Let $R_{+}^{4}=\left\{\left(x_{1}, x_{2}, x_{3}, x_{4}\right): x_{i} \geq 0, i=1,2,3,4\right\}$. Denote by $C\left([-\tau, 0], R_{+}^{4}\right)$ the Banach space of all continuous functions $\phi=\left(\phi_{1}, \phi_{2}, \phi_{3}, \phi_{4}\right):[-\tau, 0] \rightarrow R_{+}^{4}$ with the supremum norm $\|\phi\|=\sup _{-\tau \leq s \leq 0}\{|\phi(s)|\}$, where $|\phi(s)|=\sum_{i=1}^{4}\left|\phi_{i}(s)\right|$.

From the biological background of model (3), the initial condition for any solution is given by

$$
\left\{\begin{array}{l}
T(\vartheta)=\phi_{1}(\vartheta), T^{*}(\vartheta)=\phi_{2}(\vartheta), V(\vartheta)=\phi_{3}(\vartheta), B(\vartheta)=\phi_{3}(\vartheta), \\
\phi_{1}(0)>0, \phi_{2}(0)>0, \phi_{3}(0)>0, \phi_{4}(0)>0, \vartheta \in[-\tau, 0],
\end{array}\right.
$$

where $\left(\phi_{1}, \phi_{2}, \phi_{3}, \phi_{4}\right) \in C\left(\left[-\tau_{1}, 0\right], R_{+}^{4}\right)$. It is known as the basic theory of functional differential equations [43], model (3) have a unique solution $\left(T(s), T^{*}(s), V(s), B(s)\right)$ that satisfies initial conditions (4).

First of all, regarding the non-negativity and boundedness of solutions for model (3), we have the result given below.

Theorem 1 For any initial point $x_{0}=\left(T_{0}, T_{0}^{*}, V_{0}, B_{0}\right) \in R_{+}^{4 *}$, model (3) has a unique nonnegative solution $u(s)=\left(T(s), T^{*}(s), V(s), B(s)\right) \in R_{+}^{4}$ defined on $[0, \infty)$ and the solution is also ultimately bounded.

The proof of Theorem 1 is simple. In fact, the positivity of solutions can be obtained by using auxiliary function $\Gamma(s)=\min \left\{T(s), T^{*}(s), V(s), B(s)\right\}$ and reduction to absurdity. The ultimate boundedness of solutions can be verified by using the auxiliary function

$$
L(s)=T(s)+e^{m \tau_{1}} T^{*}\left(s+\tau_{1}\right)+\frac{m e^{m \tau_{1}}}{p} V\left(s+\tau_{1}\right)+\frac{m e^{m \tau_{1}} q}{\alpha p} B\left(s+\tau_{1}\right)
$$

with $f=\min \{m, d, c, \omega\}$ and the comparison principle. Therefore, we here omit it.

When $U>0$, the basic reproduction number for the antibody response virus infection for model (3) is defined by

$$
R_{w}=\frac{\alpha g(U)(m+d)(k \omega+m \alpha)+k p \omega \alpha \Lambda_{c} e^{-m \tau_{1}}}{c \omega(m+d)(k \omega+m \alpha)} .
$$

Obviously, when $U=0$ we have $R_{w}=\frac{\alpha p k \Lambda_{c} e^{-m \tau_{1}}}{c(m+d)(k \omega+m \alpha)} \triangleq R_{w 1}$.

On the existence of equilibrium for model (3), we have a result given below. 


\section{Theorem 2}

(a) When $U>0$, model (3) always has unique antibody-free infection equilibrium $A_{1}(U)=\left(T_{1}(U), T_{1}^{*}(U), V_{1}(U), 0\right)$, where $T_{1}(U), T_{1}^{*}(U)$ and $V_{1}(U)$ are given below. Furthermore, if $R_{w} \leq 1$ for some $U_{0}>0$, then for any $0<U \leq U_{0}$ model (3) only has equilibrium $A_{1}(U)$;

(b) If $R_{w}>1$ for some $U_{0}>0$, then, except for equilibrium $A_{1}(U)$, for any $U \geq U_{0}$ model (3) also has unique antibody response infection equilibrium $A_{2}(U)=\left(T_{2}(U), T_{2}^{*}(U), V_{2}(U), B_{2}(U)\right)$, where $T_{2}(U), T_{2}^{*}(U), V_{2}(U)$ and $B_{2}(U)$ are given below.

Proof Denote $R_{w 0}=\frac{k p T_{0} e^{-m \tau_{1}}}{c(m+d)}$. When $U>0$ antibody-free infection equilibrium $A_{1}(U)=\left(T_{1}(U), T_{1}^{*}(U), V_{1}(U), 0\right)$ satisfies the following equations

$$
\left\{\begin{array}{l}
\Lambda_{c}-k V_{1}(U) T_{1}(U)-m T_{1}(U)=0, \\
k V_{1}(U) T_{1}(U) e^{-m \tau_{1}}-(m+d) T_{1}^{*}(U)=0, \\
g(U)+p T_{1}^{*}(U)-c V_{1}(U)=0 .
\end{array}\right.
$$

We directly get

$$
V_{1}(U)=\frac{1}{c}\left(g(U)+p T_{1}^{*}(U)\right), \quad T_{1}^{*}(U)=\frac{m e^{-m \tau_{1}}}{m+d}\left(T_{0}-T_{1}(U)\right)
$$

and $T_{1}(U)=\frac{m}{m+k V_{1}(U)} T_{0}<T_{0}$. Substituting (6) into the first equation of (5), we further get the quadratic equation with $T_{1}(U)$ as a root

$$
T_{1}^{2}(U)-a_{1}(U) T_{1}(U)+a_{2}=0,
$$

where $a_{1}(U)=\frac{(m+d) g(U)}{p m e^{-m \tau_{1}}}+T_{0}\left(1+\frac{1}{R_{w 0}}\right), \quad a_{2}=\frac{T_{0}^{2}}{R_{w 0}}$. Since

$$
\begin{aligned}
a_{1}^{2}(U)-4 a_{2}= & {\left[\frac{(m+d) g(U)}{p m}+T_{0}\left(1+\frac{1}{R_{w 0}}\right)\right]^{2}-\frac{4 T_{0}^{2}}{R_{w 0}} } \\
& >T_{0}^{2}\left(1+\frac{1}{R_{w 0}}\right)^{2}-\frac{4 T_{0}^{2}}{R_{w 0}} \geq 0
\end{aligned}
$$

equation (7) has two positive roots as follows

$$
T_{1 \pm}(U)=\frac{1}{2}\left(a_{1}(U) \pm \sqrt{a_{1}^{2}(U)-4 a_{2}}\right)
$$

Computing the derivative of $T_{1 \pm}(U)$ we have $T_{1 \pm}^{\prime}(U)=\frac{1}{2} a_{1}^{\prime}(U)\left(1 \pm \frac{a_{1}}{\sqrt{a_{1}^{2}-4 a_{2}}}\right)$. Because of $a_{2}>0$ and $a_{1}^{\prime}(U)>0$, we further have $T_{+}^{\prime}(U)>0$ and $T_{-}^{\prime}(U)<0$ for 
$U>0$. From (8) we can obtain

$$
T_{1+}(0)=\left\{\begin{aligned}
T_{0}, & \text { if } R_{w 0} \geq 1, \\
\frac{T_{0}}{R_{w 0}}, & \text { if } R_{w 0}<1
\end{aligned}\right.
$$

and

$$
T_{1-}(0)=\left\{\begin{aligned}
\frac{T_{0}}{R_{w 0}}, & \text { if } R_{w 0}>1 \\
T_{0}, & \text { if } R_{w 0} \leq 1
\end{aligned}\right.
$$

Since $T_{1+}(U) \geq T_{1+}(0) \geq T_{0}$ and $T_{1-}(U) \leq T_{1-}(0) \leq T_{0}$ for any $U>0$, we can get that model (3) has unique antibody-free infection equilibrium $A_{1}(U)=$ $\left(T_{1}(U), T_{1}^{*}(U), V_{1}(U), 0\right)$ with

$$
\begin{aligned}
& T_{1}(U)=\frac{1}{2}\left(a_{1}(U)-\sqrt{a_{1}^{2}(U)-4 a_{2}}\right)=\frac{T_{0}}{R_{v}(U)}, \quad T_{1}^{*}(U)=\frac{\Lambda_{c} e^{-m \tau_{1}}}{m+d}\left(1-\frac{1}{R_{v}(U)}\right) \\
& V_{1}(U)=\frac{1}{c}\left(g(U)+\frac{p \Lambda_{c} e^{-m \tau_{1}}}{m+d}\left(1-\frac{1}{R_{v}(U)}\right)\right), \quad R_{v}(U)=\frac{2 T_{0}}{a_{1}(U)-\sqrt{a_{1}^{2}(U)-4 a_{2}}} .
\end{aligned}
$$

We have that antibody response infection equilibrium $A_{2}(U)=\left(T_{2}(U), T_{2}^{*}(U)\right.$, $\left.V_{2}(U), B_{2}(U)\right)$ satisfies the following equations

$$
\left\{\begin{array}{l}
\Lambda_{c}-k V_{2}(U) T_{2}(U)-m T_{2}(U)=0 \\
k V_{2}(U) T_{2}(U) e^{-m \tau_{1}}-(m+d) T_{2}^{*}(U)=0 \\
g(U(t))+p T_{2}^{*}(U)-c V_{2}(U)-q B_{2}(U) V_{2}(U)=0 \\
\alpha B_{2}(U) V_{2}(U)-\omega B_{2}(U)=0
\end{array}\right.
$$

by calculating we directly can obtain

$$
\begin{aligned}
& V_{2}(U)=\frac{\omega}{\alpha}, \quad T_{2}^{*}(U)=\frac{k \omega \Lambda_{c} e^{-m \tau_{1}}}{(m+d)(k \omega+m \alpha)}, \quad T_{2}(U)=\frac{\Lambda_{c} \alpha}{k \omega+m \alpha} \\
& B_{2}(U)=\frac{\alpha g(U)(m+d)(k \omega+m \alpha)+k p \omega \alpha \Lambda_{c} e^{-m \tau_{1}}-c \omega(m+d)(k \omega+m \alpha)}{q \omega(m+d)(k \omega+m \alpha)} .
\end{aligned}
$$

Obviously, if $R_{w} \leq 1$ for some $U_{0}>0$, then $B_{2}(U) \leq 0$ for any $0<U \leq U_{0}$, which implies that model (3) only has equilibrium $A_{1}(U)$. If $R_{w}>1$ for some $U_{0}>0$, then $B_{2}(U)>0$ for any $U \geq U_{0}$, which implies that model (3) also has unique equilibrium $A_{2}(U)$. This completes the proof.

\subsection{Stability of equilibria}

Firstly, we proved the stability of antibody-free infection equilibrium $A_{1}(U)=$ $\left(T_{1}(U), T_{1}^{*}(U), V_{1}(U), 0\right)$ with $U>0$. We get a conclusion given below. 
Theorem 3 Assume $R_{w} \leq 1$ for some $U_{0}>0$. Then for any $0<U \leq U_{0}$ equilibrium $A_{1}(U)$ is globally asymptotically stable.

Proof The defined Lyapunov function $L_{1}$ is as follows

$$
\begin{aligned}
L_{1}= & e^{-m \tau_{1}} T_{1}\left(\frac{T}{T_{1}}-\ln \frac{T}{T_{1}}-1\right)+T_{1}^{*}\left(\frac{T^{*}}{T_{1}^{*}}-\ln \frac{T^{*}}{T_{1}^{*}}-1\right)+e^{-m \tau_{1}} k V_{1} T_{1} \int_{s-\tau_{1}}^{s} \\
& \left(\frac{k V T}{k V_{1} T_{1}}-1-\ln \frac{k V T}{k V_{1} T_{1}}\right) d x+\frac{m+d}{p} V_{1}\left(\frac{V}{V_{1}}-\ln \frac{V}{V_{1}}-1\right)+\frac{m+d}{\alpha p} q B .
\end{aligned}
$$

The derivative of $L_{1}$ along with solutions of model (3) is given by

$$
\begin{aligned}
& \frac{d L_{1}}{d t}=e^{-m \tau_{1}}\left(1-\frac{T_{1}}{T}\right)\left(k V_{1} T_{1}+m T_{1}-k V T-m T\right)+\left(1-\frac{T_{1}^{*}}{T^{*}}\right)\left(k V\left(s-\tau_{1}\right)\right. \\
& \left.\times T\left(s-\tau_{1}\right) e^{-m \tau_{1}}-(m+d) T^{*}\right)+e^{-m \tau_{1}} k V_{1} T_{1}\left(\frac{k V(s) T(s)}{k V_{1} T_{1}}\right. \\
& \left.-\ln \frac{k V(s) T(s)}{k V_{1} T_{1}}-\frac{k V\left(s-\tau_{1}\right) T\left(s-\tau_{1}\right)}{k V_{1} T_{1}}+\ln \frac{k V\left(s-\tau_{1}\right) T\left(s-\tau_{1}\right)}{k V_{1} T_{1}}\right) \\
& +\frac{m+d}{p}\left(1-\frac{V_{1}}{V}\right)\left(p T^{*}-c V-q B V\right)+\frac{q(m+d)}{\alpha p}(\alpha B V-\omega B) \\
& =e^{-m \tau_{1}} m T_{1}\left(2-\frac{T}{T_{1}}-\frac{T_{1}}{T}\right)+e^{-m \tau_{1}} k V_{1} T_{1}-e^{-m \tau_{1}} \frac{T_{1}}{T} k V_{1} T_{1} \\
& -e^{-m \tau_{1}} k V\left(s-\tau_{1}\right) T\left(s-\tau_{1}\right) \frac{T_{1}^{*}}{T^{*}}+(m+d) T_{1}^{*}-(m+d) T^{*} \frac{V_{1}}{V} \\
& +\frac{(m+d) g(U)}{p}-\frac{(m+d) g(U)}{p} \frac{V_{1}}{V}+\frac{(m+d) g(U)}{p}-\frac{(m+d) g(U)}{p} \frac{V}{V_{1}} \\
& +\frac{m+d}{p} q B V_{1}-\frac{m+d}{\alpha p} q \omega B+(m+d) T_{1}^{*}-\frac{m+d}{p} B_{1} V_{1}+\frac{m+d}{p} B_{1} V \\
& -e^{-m \tau_{1}} k V_{1} T_{1} \ln \frac{k V(s) T(s)}{k V_{1} T_{1}}+e^{-m \tau_{1}} k V_{1} T_{1} \ln \frac{k V\left(s-\tau_{1}\right) T\left(s-\tau_{1}\right)}{k V_{1} T_{1}} \\
& =e^{-m \tau_{1}} m T_{1}\left(2-\frac{T}{T_{1}}-\frac{T_{1}}{T}\right)+\frac{(m+d) g(U)}{p}\left(2-\frac{V}{V_{1}}-\frac{V_{1}}{V}\right) \\
& -e^{-m \tau_{1}} k V_{1} T_{1}\left(\frac{k V\left(s-\tau_{1}\right) T\left(s-\tau_{1}\right)}{k V_{1} T_{1}} \frac{T_{1}^{*}}{T^{*}}-1-\ln \frac{k V\left(s-\tau_{1}\right) T\left(s-\tau_{1}\right)}{k V_{1} T_{1}} \frac{T_{1}^{*}}{T^{*}}\right) \\
& -e^{-m \tau_{1}} k V_{1} T_{1}\left(\frac{T^{*} V_{1}}{T_{1}^{*} V}-1-\ln \frac{T^{*} V_{1}}{T_{1}^{*} V}\right)-e^{-m \tau_{1}} k V_{1} T_{1}\left(\frac{T_{1}}{T}-1-\ln \frac{T_{1}}{T}\right) \\
& +\frac{q(m+d)}{p}\left(V_{1}-\frac{\omega}{\alpha}\right) B \text {. }
\end{aligned}
$$

Obviously, by calculating we can get $V_{1} \leq \frac{\omega}{\alpha}$ when $R_{w} \leq 1$. Hence, for any $T(s), T^{*}(s), V(s), B(s)$ we have $\frac{d L_{1}}{d t} \leq 0$. Moreover, we have $\frac{d L_{1}}{d t}=0$ if and only if $\left(T(s), T^{*}(s), V(s), B(s)\right)=\left(T_{1}(s), T_{1}^{*}(s), V_{1}(s), B_{1}(s)\right)$. According to the LaSalle's invariance principle, clearly we have equilibrium $A_{1}(U)$ for model (3) is globally asymptotically stable. 
Theorem 4 Assume $R_{w}>1$ for some $U_{0}>0$. Then for any $U \geq U_{0}$ equilibrium $A_{1}(U)$ is unstable and $A_{2}(U)$ is local asymptotically stable.

Proof For the convenience of calculation, we denote $\left(T_{1}(U), T_{1}^{*}(U), V_{1}(U), 0\right)=$ $\left(T_{1}, T_{1}^{*}, V_{1}, 0\right)$ and $\left(T_{2}(U), T_{2}^{*}(U), V_{2}(U), B_{2}(U)\right)=\left(T_{2}, T_{2}^{*}, V_{2}, B_{2}\right)$. Firstly, by the detailed calculation the linearized system of model (3) at any equilibrium $\bar{A}=$ $\left(\bar{T}, T^{*}, \bar{V}, \bar{B}\right)$ can be obtained as follows

$$
\left\{\begin{array}{l}
\frac{d x(s)}{d s}=-k z(s) \bar{T}-k \bar{V} x(s)-m x(s), \\
\frac{d y(s)}{d s}=k e^{-m \tau_{1}} z\left(s-\tau_{1}\right) \bar{T}+k e^{-m \tau_{1}} x\left(s-\tau_{1}\right) \bar{V}-(m+d) y(s), \\
\frac{d z(s)}{d s}=p y(s)-c z(s)-q h(s) \bar{V}-q \bar{B} z(s), \\
\frac{d h(s)}{d s}=\alpha h(s) \bar{V}+\alpha \bar{B} z(s)-\omega h(s)
\end{array}\right.
$$

From system (13) we further obtain the characteristic equation at equilibrium $\bar{A}=$ $\left(\bar{T}, \bar{T}^{*}, \bar{V}, \bar{B}\right)$ as follows

$$
A(\lambda)=\left|\begin{array}{cccc}
\lambda+k \bar{V}+m & 0 & k \bar{T} & 0 \\
-k \bar{V} e^{-(\lambda+m) \tau_{1}} & \lambda+m+d & -k \bar{T} e^{-(\lambda+m) \tau_{1}} & 0 \\
0 & -p & \lambda+c+q \bar{B} & q \bar{V} \\
0 & 0 & -\alpha \bar{B} & (\lambda+\omega-\alpha \bar{V})
\end{array}\right|=0
$$

According to (14), the characteristic equation of model (3) at equilibrium $A_{1}(U)$ is

$$
\begin{aligned}
A(\lambda)= & \left(\lambda+\omega-\alpha V_{1}\right)\left[\lambda^{3}+\lambda^{2}\left(c+m+d+m+k V_{1}\right)+\lambda\left(\left(m+d+m+k V_{1}\right) c\right.\right. \\
& \left.+(m+d)\left(m+k V_{1}\right)\right)+c(m+d)\left(m+k V_{1}\right) \\
& \left.+\left(-\lambda p k T_{1} e^{-m \tau_{1}}-p k T_{1} m e^{-m \tau_{1}}\right) e^{-\lambda \tau_{1}}\right]=0 .
\end{aligned}
$$

Obviously, equation (15) has a root $\lambda=\alpha V_{1}-\omega$. When $R_{w}>1$, we have

$$
\begin{aligned}
\lambda & =\alpha V_{1}-\omega=\frac{\alpha p \Lambda_{c} e^{-m \tau_{1}}\left(1-\frac{1}{R_{v}(U)}\right)+\alpha g(U)(m+d)-\omega c(m+d)}{c(m+d)} \\
& =\frac{\alpha p \Lambda_{c} e^{-m \tau_{1}}\left(1-\frac{1}{R_{v}(U)}\right)(k \omega+m \alpha)+(\alpha g(U)-\omega c)(m+d)(k \omega+m \alpha)}{c(m+d)(k \omega+m \alpha)} \\
& =\frac{\omega c(m+d)(k \omega+m \alpha)\left(R_{w}-1\right)+\alpha p \Lambda_{c} e^{-m \tau_{1}}\left(m \alpha-\frac{1}{R_{v}(U)}(k \omega+m \alpha)\right)}{c(m+d)(k \omega+m \alpha)} .
\end{aligned}
$$

We can have $\lambda>0$, and this implies that equilibrium $A_{1}(U)$ is unstable for any $\tau_{1} \geq 0$. 
Next, from (14) the characteristic equation of model (3) at equilibrium $A_{2}(U)$ is

$$
\begin{aligned}
A(\lambda)= & \left|\begin{array}{cccc}
\lambda+k V_{2}+m & 0 & k T_{2} & 0 \\
-k V_{2} e^{-(\lambda+m) \tau_{1}} & \lambda+m+d-k T_{2} e^{-(\lambda+m) \tau_{1}} & 0 \\
0 & -p & \lambda+c+q B_{2} & q V_{2} \\
0 & 0 & -\alpha B_{2} & \left(\lambda+\omega-\alpha V_{2}\right)
\end{array}\right|=0 . \\
= & \left(\lambda+k V_{2}+m\right)(\lambda+m+d)\left[\left(\lambda+c+q B_{2}\right)\left(\lambda+\omega-\alpha V_{2}\right)\right. \\
& \left.+q V_{2} \alpha B_{2}\right]+\left(\lambda+\omega-\alpha V_{2}\right)\left[p k T_{2}(U) k V_{2} e^{-(\lambda+m) \tau_{1}}\right. \\
& \left.-\left(\lambda+k V_{2}+m\right) p k T_{2} e^{-(\lambda+m) \tau_{1}}\right]=0 .
\end{aligned}
$$

Furthermore, the characteristic equation becomes into the following form

$$
\begin{aligned}
& \left(\lambda+k V_{2}+m\right)(\lambda+m+d)\left[\left(\lambda+c+q B_{2}\right)\left(\lambda+\omega-\alpha V_{2}\right)+q V_{2} \alpha B_{2}\right] \\
& \quad=\left(\lambda+\omega-\alpha V_{2}\right)(\lambda+m) p k T_{2} e^{-(\lambda+m) \tau_{1}} .
\end{aligned}
$$

Suppose that (16) has a root $\lambda=\rho+i \omega_{0}$ with $\rho \geq 0$ and $\omega_{0} \geq 0$, which implies $\left|e^{-\lambda \tau_{1}}\right|<1$. Denote

$$
\begin{aligned}
L H S= & \left(\rho+i \omega_{0}+k V_{2}+m\right)\left(\rho+i \omega_{0}+m+d\right)\left[\left(\rho+i \omega_{0}+c R_{w}\right)\left(\rho+i \omega_{0}\right)\right. \\
& \left.+q V_{2} \alpha B_{2}\right], \\
\text { RHS }= & \left(\rho+i \omega_{0}\right)\left(\rho+i \omega_{0}+m\right) p k T_{2} e^{-(\lambda+m) \tau_{1}} .
\end{aligned}
$$

Since

$$
\left|q V_{2} \alpha B_{2}+\left(\rho+i \omega_{0}+c+q B_{2}\right)\left(\rho+i \omega_{0}\right)\right|>\left|\left(\rho+i \omega_{0}\right) c R_{w}\right|
$$

and

$$
\left|\rho+i \omega_{0}+k V_{2}+m\right|>\left|\rho+i \omega_{0}+m\right|,\left|\rho+i \omega_{0}+m+d\right|>(m+d),
$$

we further have

$$
\begin{aligned}
|L H S| & =\left|\left(\rho+i \omega_{0}+k V_{2}+m\right)\right|\left|\left(\rho+i \omega_{0}+m+d\right)\right| \\
& \times\left|\left(\rho+i \omega_{0}+c R_{w}\right)\left(\rho+i \omega_{0}\right)+q V_{2} \alpha B_{2}\right| \\
> & \left|\left(\rho+i \omega_{0}+m\right)(m+d)\left(\rho+i \omega_{0}\right) c R_{w}\right| .
\end{aligned}
$$


Since

$$
\begin{aligned}
p k T_{2} e^{-m \tau} e^{-\lambda \tau_{1}}= & c(m+d)\left[\frac{\alpha g(U)(m+d)(k \omega+m \alpha)+k p \omega \alpha \Lambda_{c} e^{-m \tau_{1}}}{c \omega(m+d)(k \omega+m \alpha)}\right. \\
& \left.-\frac{\alpha g(U)(m+d)(k \omega+m \alpha)}{c \omega(m+d)(k \omega+m \alpha)}\right] e^{-\lambda \tau_{1}} \\
< & c(m+d) \frac{\alpha g(U)(m+d)(k \omega+m \alpha)+k p \omega \alpha \Lambda_{c} e^{-m \tau_{1}}}{c \omega(m+d)(k \omega+m \alpha)} \\
= & (m+d) c R_{w},
\end{aligned}
$$

then we have

$$
\begin{aligned}
|R H S| & =\left|\left(\rho+i \omega_{0}\right)\right|\left|\left(\rho+i \omega_{0}+m\right)\right|\left|p k T_{2}(U) e^{-(\lambda+m) \tau_{1}}\right| \\
& <\left|\left(\rho+i \omega_{0}+m\right)(m+d)\left(\rho+i \omega_{0}\right) c R_{w}\right| .
\end{aligned}
$$

From the above calculations, we can easily see that the modulus on the left-hand side of expression (17) is greater than the modulus on the right-hand side. This is an obvious contradiction. Therefore, for any $\tau_{1} \geq 0$, equation (16) will not have roots with non-negative real parts. Therefore, we can determine that the equilibrium point $A_{2}(U)$ is locally asymptotically stable.

Remark 1 Clearly know that the local stability of equilibrium $A_{1}(U)$ is simple, so in Theorem 3 we directly give the global stability for $A_{1}(U)$. The global stability for $A_{2}(U)$ has not been proved yet, so in Theorem 4 we only obtain that $A_{1}(U)$ is unstable and $A_{2}(U)$ is local asymptotically stable. Therefore, an interesting open problem is to prove that $A_{2}(U)$ is globally asymptotically stable when $R_{w}>1$.

Remark 2 From the main conclusions obtained in this section, we easily find that when there is the environmental virus unremitting invading then the virus infection in host always occurs.

Remark 3 From the basic reproduction number $R_{w}$, we easily see that $R_{w}$ is decreasing when latent delay $\tau_{1}$ increases. This shows that, due to there is not virus infection in latent period, the long latent period can retard the outbreak of virus infection in host.

Remark 4 From Theorem 4 we see that when there is antibody immunity response in host then the virus in host will ultimately reach a steady numerical value $\frac{\omega}{\alpha}$, where $\alpha$ indicates the production rate of $B$ cells, $\omega$ indicates the mortality rate of $B$ cells. Therefore, increasing the recruitment rate of $B$ cells will decrease the numerical value of virus, and then retard the virus infection in host.

\section{Disease transmission model with incubation delay and immune response in host}

In this part, we further study the disease transmission model (2). It is clear that for the infection disease caused by the virus infection, the process of virus infection in 
host is very quick than the speed of disease transmission. Thence, we can propose the following reasonable assumption.

$\left(\mathbf{H}_{2}\right)$ In the process of disease transmission, the virus infection in host always achieve its steady state.

When the infection disease is prevalent, we know that there must be virus infection in host. Therefore, we always have $U>0$. Further, we consider that there is the immune response in host, then we also have $R_{w}>1$ for any $U>0$. Obviously, when $R_{w 1}>1$ then $R_{w}>1$ for any $U>0$. Thus, based on the discussions in above section and due to the local stability of equilibrium $A_{2}(U)=\left(T_{2}(U), T_{2}^{*}(U), V_{2}(U), B_{2}(U)\right)$ from Theorem 4, we can assume that in host virus $V(s) \rightarrow V_{2}(U)=\frac{\omega}{\alpha}$ as $s \rightarrow \infty$. Therefore, based on assumption $\left(\mathbf{H}_{2}\right)$ we can replace $V=V_{2}(U)=\frac{\omega}{\alpha}$ in disease transmission model (2), and further obtain the following model

$$
\left\{\begin{array}{l}
\frac{d S}{d t}=\Lambda-\beta_{1} U(t) S(t)-\beta_{2} I(t) S(t)-\mu S(t), \\
\frac{d I}{d t}=\left(\beta_{1} U(t-\tau) S(t-\tau)+\beta_{2} I(t-\tau) S(t-\tau)\right) e^{-\mu \tau}-(\mu+\sigma+\zeta) I(t), \\
\frac{d R}{d t}=\sigma I(t)-(\mu+\delta) R(t), \\
\frac{d U}{d t}=\theta I(t) \frac{\omega}{\alpha}-(\xi+\gamma) U(t) .
\end{array}\right.
$$

The initial conditions for model (18) are given by

$$
\left\{\begin{array}{l}
S(v)=\phi_{1}(v), I(v)=\phi_{2}(v), R(v)=\phi_{3}(v), U(v)=\phi_{4}(v), \\
\phi_{1}(0)>0, \phi_{2}(0)>0, \phi_{3}(0)>0, \phi_{4}(0)>0, v \in[-\tau, 0]
\end{array}\right.
$$

where $\phi=\left(\phi_{1}, \phi_{2}, \phi_{3}, \phi_{4}\right) \in C\left([-\tau, 0], R_{+}^{4}\right)$.

Since the variable $R$ in model (18) is not included in other three equations, it is sufficient that we only need to study the following 3-dimensional model

$$
\left\{\begin{array}{l}
\frac{d S}{d t}=\Lambda-\beta_{1} U(t) S(t)-\beta_{2} I(t) S(t)-\mu S(t), \\
\frac{d I}{d t}=\left(\beta_{1} U(t-\tau) S(t-\tau)+\beta_{2} I(t-\tau) S(t-\tau)\right) e^{-\mu \tau}-(\mu+\sigma+\zeta) I(t), \\
\frac{d U}{d t}=\theta I(t) \frac{\omega}{\alpha}-(\xi+\gamma) U(t) .
\end{array}\right.
$$

\subsection{Basic properties}

Theorem 5 The solution $(S(t), I(t), R(t), U(t))$ of model (18) satisfying initial condition (19) is defined for all $t \geq 0$ and is nonnegative and ultimately bounded. 
The non-negativity of solution is easy to prove. Furthermore, using the auxiliary function

$$
N(t)=S(t)+e^{\mu \tau} I(t+\tau)+R(t+\tau) e^{\mu \tau}
$$

with $f=\min \{\mu, \mu+\zeta, \mu+\zeta\}$, we can easily imply the ultimate boundedness of solutions. Here, we omit the detailed proof.

In general, we use $R_{0}$ to represent the corresponding basic reproduction number. We can define it as the number of people infected by a patient during the average disease period when all individuals are susceptible at the initial stage of the disease [44]. For model (20), we have calculated the corresponding basic reproduction number

$$
R_{0}=\frac{\left(\beta_{2} \Lambda(\xi+\gamma)+\beta_{1} \theta V \Lambda\right) e^{-\mu \tau}}{\mu(\mu+\sigma+\zeta)(\xi+\gamma)},
$$

where, for the convenience of calculation, we denote $V=\frac{\omega}{\alpha}$.

Denote $S_{0}=\frac{\Lambda}{\mu}$. Let

$$
R_{0}^{(1)}=\frac{\beta_{1} \theta \omega S_{0} e^{-\mu \tau}}{\alpha(\mu+\sigma+\zeta)(\xi+\gamma)}, R_{0}^{(2)}=\frac{\beta_{2} S_{0} e^{-\mu \tau}}{\mu+\sigma+\zeta}
$$

Obviously, we see that $R_{0}^{(1)}$ is the basic reproduction number for the indirect transmission of the disease driven by the environment, while $R_{0}^{(2)}$ is the basic reproduction number for the direct transmission of the disease by the infected individual. Then we have

$$
R_{0}=R_{0}^{(1)}+R_{0}^{(2)}
$$

namely, the total basic reproduction number $R_{0}$ of model (20) is the sum of indirect basic reproduction number $R_{0}^{(1)}$ and direct basic reproduction number $R_{0}^{(2)}$

Remark 5 According to the biological definition of all parameters in model (18), and expressions for the indirect and direct basic reproduction numbers $R_{0}^{(1)}$ and $R_{0}^{(2)}$. It is apparent to us that $R_{0}^{(1)}$ is increasing with respect to the infection rate $\beta_{1}$ of susceptible in infected environment, the emission rate $\theta$ of virus to the environment released by each infected individual and the mortality rate $\omega$ of $B$ cells. $R_{0}^{(1)}$ is decreasing with respect to the production rate $\alpha$ of $B$ cells, the incubation period $\tau$, the clearance rate $\gamma$ of environmental virus and the quarantine and cure rate $\sigma$ of infected individuals. $R_{0}^{(2)}$ is increasing with respect to the infection rate $\beta_{2}$ of susceptible infected by infected individuals. $R_{0}^{(2)}$ is decreasing with respect to the mortality of virus in the environment, the incubation period $\tau$ and the cure rate $\sigma$ of infected individuals.

The above discussions tell us that the total basic reproduction number $R_{0}$ is increasing with respect to the infection rate $\beta_{1}$ of susceptible in infected environment, the emission rate $\theta$ of virus to the environment released by each infected individual, the mortality rate $\omega$ of $B$ cells, the infection rate $\beta_{2}$ of susceptible infected by infected 
individuals. $R_{0}$ is decreasing with respect to the production rate $\alpha$ of $B$ cells, the clearance rate $\gamma$ of environmental virus, the incubation period $\tau$ and the quarantine and cure rate $\sigma$ of infected individuals.

Apparently, model (20) has a disease-free equilibrium $E_{0}=\left(S_{0}, 0,0\right)$. On the existence of endemic equilibrium $E^{*}$ we easily come to the following conclusion.

\section{Theorem 6}

(a) When $R_{0} \leq 1$, model (20) has only disease-free equilibrium $E_{0}=\left(S_{0}, 0,0\right)$.

(b) When $R_{0}>1$, model (20) has unique endemic equilibrium $E^{*}=\left(S^{*}, I^{*}, U^{*}\right)$, where

$$
S^{*}=\frac{S_{0}}{R_{0}}, I^{*}=\frac{(\xi+\gamma) \mu\left(R_{0}-1\right)}{\beta_{1} \theta V+\beta_{2}(\xi+\gamma)}, U^{*}=\frac{\theta V \mu\left(R_{0}-1\right)}{\beta_{1} \theta V+\beta_{2}(\xi+\gamma)}
$$

\subsection{Stability of equilibria}

Now, we prove the stability of equilibria $E_{0}$ and $E^{*}$. Let $\tilde{E}=(\tilde{S}, \tilde{I}, \tilde{U})$ be any equilibrium of model (20). We firstly calculate the linearized system of model (20) at equilibrium $\tilde{E}$ as follows.

$$
\left\{\begin{aligned}
\frac{d x(t)}{d t}= & -\beta_{1} z(t) \tilde{S}-\beta_{1} x(t) \tilde{U}-\beta_{2} y(t) \tilde{S}-\beta_{2} \tilde{I} x(t)-\mu x(t), \\
\frac{d y(t)}{d t}= & \beta_{1} e^{-\mu \tau} z(t-\tau) \tilde{S}+\beta_{1} e^{-\mu \tau} \tilde{U} x(t-\tau)+\beta_{2} \tilde{I} x(t-\tau) e^{-\mu \tau} \\
& +\beta_{2} y(t-\tau) \tilde{S} e^{-\mu \tau}-(\mu+\sigma+\zeta) y(t), \\
\frac{d z(t)}{d t}= & \theta y(t) V-(\xi+\gamma) z(t) .
\end{aligned}\right.
$$

From this, we further can get the characteristic equation of model (20) at any equilibrium $\tilde{E}$ as follows.

$$
Q(\lambda)=\left|\begin{array}{ccc}
\lambda+a_{11} & \beta_{2} \tilde{S} & \beta_{1} \tilde{S} \\
a_{21} & \lambda-a_{22} & a_{23} \\
0 & -\theta V & \lambda+(\xi+\gamma)
\end{array}\right|=0
$$

where $a_{11}=\beta_{1} \tilde{U}+\beta_{2} \tilde{I}+\mu, a_{21}=-\beta_{1} \tilde{U} e^{-(\lambda+\mu) \tau}-\beta_{2} \tilde{I} e^{-(\lambda+\mu) \tau}, a_{22}=$ $\beta_{2} e^{-(\lambda+\mu) \tau} \tilde{S}+\mu+\sigma+\zeta$ and $a_{23}=-\beta_{1} \tilde{S} e^{-(\lambda+\mu) \tau}$.

We are now in a position to study the local stability of disease-free equilibrium $E_{0}$. We have the following result.

\section{Theorem 7}

(i) If $R_{0} \leq 1$, then equilibrium $E_{0}$ is locally asymptotically stable.

(ii) If $R_{0}>1$, then $E_{0}$ is unstable. 
Proof According to (23), we know that the characteristic equation of model (20) at equilibrium $E_{0}$ is

$$
\begin{aligned}
Q(\lambda)= & (\lambda+\mu)\left(\lambda^{2}+\lambda\left(\mu+\sigma+\zeta-\beta_{2} e^{-(\lambda+\mu) \tau} \frac{\Lambda}{\mu}+\xi+\gamma\right)\right. \\
& \left.+\left(\mu+\sigma+\zeta-\beta_{2} e^{-(\lambda+\mu) \tau} \frac{\Lambda}{\mu}\right)(\xi+\gamma)-\beta_{1} \theta V \frac{\Lambda}{\mu} e^{-\tau(\lambda+\mu)}\right)=0 .
\end{aligned}
$$

When $\tau=0$, equation (24) becomes into the following form

$$
(\lambda+\mu)\left(\lambda^{2}+\lambda\left(\mu+\sigma+\zeta-\beta_{2} \frac{\Lambda}{\mu}+\xi+\gamma\right)+\left(\mu+\sigma+\zeta-\beta_{2} \frac{\Lambda}{\mu}\right)(\xi+\gamma)-\beta_{1} \theta V \frac{\Lambda}{\mu}\right)=0
$$

Clearly, we can see that equation (25) has one negative root $\lambda_{1}=-\mu$. For the other two roots $\lambda_{2}$ and $\lambda_{3}$ of equation (25), we have

$$
\begin{aligned}
\lambda_{2}+\lambda_{3}= & -\left(\mu+\sigma+\zeta-\beta_{2} \frac{\Lambda}{\mu}+\xi+\gamma\right)=-\left[(\mu+\sigma+\zeta)\left(1-\frac{\beta_{2} \Lambda}{\mu(\mu+\sigma+\zeta)}\right)\right. \\
& +(\xi+\gamma)]<0 \\
\lambda_{2} \lambda_{3}= & (\xi+\gamma)(\mu+\sigma+\zeta)\left(1-\frac{\beta_{2} \Lambda(\xi+\gamma) e^{-\mu \tau}+\beta_{1} \theta V \Lambda e^{-\mu \tau}}{\mu(\mu+\sigma+\zeta)(\xi+\gamma)}\right)>0
\end{aligned}
$$

with $R_{0}<1$. Hence, $\lambda_{2}$ and $\lambda_{3}$ have negative real parts.

Let $\tau>0$, equation (24) becomes into the following form

$$
Q(\lambda)=\lambda^{2}+a_{1} \lambda+a_{0}-\left(b_{1} \lambda+b_{0}\right) e^{-\lambda \tau}=0,
$$

where $a_{1}=(\mu+\sigma+\zeta+\xi+\gamma), a_{0}=(\xi+\gamma)(\mu+\sigma+\zeta), b_{1}=\beta_{2} \frac{\Lambda}{\mu} e^{-\mu \tau}$ and $b_{0}=(\xi+\gamma) \beta_{2} \frac{\Lambda}{\mu} e^{-\mu \tau}+\beta_{1} \theta V \frac{\Lambda}{\mu} e^{-\mu \tau}$.

Assume that $\lambda=\omega i(\omega>0)$ is a pure imaginary root of equation (26). Substitute it into equation (26) and separate the imaginary and real parts by using $e^{-\lambda \tau}=$ $\cos (\omega \tau)-i \sin (\omega \tau)$, we can get

$$
\left\{\begin{aligned}
-\omega^{2}+a_{0} & =\omega b_{1} \sin (\omega \tau)+b_{0} \cos (\omega \tau) \\
\omega a_{1} & =\omega b_{1} \cos (\omega \tau)-b_{0} \sin (\omega \tau)
\end{aligned}\right.
$$

We add the squares of (27) to get

$$
\omega^{4}+p \omega^{2}+q=0,
$$


where $p=a_{1}^{2}-2 a_{0}-b_{1}^{2}$ and $q=a_{0}^{2}-b_{0}^{2}=\left(a_{0}+b_{0}\right)\left(a_{0}-b_{0}\right)$. Since

$$
\begin{aligned}
p= & a_{1}^{2}-2 a_{0}-b_{1}^{2}=(\mu+\sigma+\zeta)^{2}\left(1-\left[\frac{\beta_{2} \Lambda e^{-\mu \tau}}{\mu(\mu+\sigma+\zeta)}\right]^{2}\right)+(\xi+\gamma)^{2}, \\
q= & a_{0}^{2}-b_{0}^{2}=\left((\xi+\gamma)(\mu+\sigma+\zeta)+(\xi+\gamma) \beta_{2} \frac{\Lambda}{\mu} e^{-\mu \tau}+\beta_{1} \theta V \frac{\Lambda}{\mu} e^{-\mu \tau}\right) \\
& \times(\xi+\gamma)(\mu+\sigma+\zeta)\left[1-\frac{\beta_{2} \Lambda(\xi+\gamma) e^{-\mu \tau}+\beta_{1} \theta V \Lambda e^{-\mu \tau}}{\mu(\mu+\sigma+\zeta)(\xi+\gamma)}\right],
\end{aligned}
$$

when $R_{0} \leq 1$ we have that $p$ and $q$ are non-negative. This shows that equation (28) has not any positive root $\omega$, which leads an obvious contradiction. Hence, we finally know when $R_{0}<1$ all roots of characteristic equation (24) have the negative real parts for any $\tau>0$. This means that the disease-free equilibrium $E_{0}$ is locally asymptotically stable.

Theorem 8 If $R_{0}>1$, then endemic equilibrium $E^{*}$ of model (20) is local asymptotically stable.

Proof From equation (23) we can get that the characteristic equation for model (20) at endemic equilibrium $E^{*}$ is given as follows

$$
\begin{aligned}
H(\lambda)= & \left(\lambda+\beta_{1} U^{*}+\beta_{2} I^{*}+\mu\right)\left[\left(\lambda-\beta_{2} e^{-(\lambda+\mu) \tau} S^{*}+\mu+\sigma+\zeta\right)(\lambda+\xi+\gamma)\right. \\
& \left.-\theta V \beta_{1} S^{*} e^{-(\lambda+\mu) \tau}\right]+\left(\beta_{1} U^{*} e^{-(\lambda+\mu) \tau}+\beta_{2} I^{*} e^{-(\lambda+\mu) \tau}\right) \\
& \times\left[\beta_{2} S^{*}(\lambda+\xi+\gamma)+\beta_{1} S^{*} \theta V\right] \\
= & \lambda^{3}+a_{2} \lambda^{2}+a_{1} \lambda+a_{0}-\left(b_{2} \lambda^{2}+b_{1} \lambda+b_{0}\right) e^{-\lambda \tau}=0,
\end{aligned}
$$

where

$$
\begin{aligned}
& a_{2}=(\mu+\sigma+\zeta)+\left(\beta_{1} U^{*}+\beta_{2} I^{*}+\mu\right)+(\xi+\gamma) \\
& a_{1}=(\mu+\sigma+\zeta)(\xi+\gamma)+(\mu+\sigma+\zeta+\xi+\gamma)\left(\beta_{1} U^{*}+\beta_{2} I^{*}+\mu\right) \\
& a_{0}=(\mu+\sigma+\zeta)\left(\beta_{1} U^{*}+\beta_{2} I^{*}+\mu\right)(\xi+\gamma), b_{2}=\beta_{2} S^{*} e^{-\mu \tau} \\
& b_{1}=\beta_{2} S^{*}(\xi+\gamma) e^{-\mu \tau}+\theta V \beta_{1} S^{*} e^{-\mu \tau}+\mu \beta_{2} S^{*} e^{-\mu \tau} \\
& b_{0}=(\xi+\gamma) \mu e^{-\mu \tau} \beta_{2} S^{*}+\theta V \mu \beta_{1} S^{*} e^{-\mu \tau} .
\end{aligned}
$$

When $\tau=0$, equation (29) becomes into the following form

$$
\lambda^{3}+\left(a_{2}-b_{2}\right) \lambda^{2}+\left(a_{1}-b_{1}\right) \lambda+a_{0}-b_{0}=0 .
$$


By calculating, we can obtain

$$
\begin{aligned}
p_{2}= & a_{2}-b_{2} \\
= & (\mu+\sigma+\zeta)+\left(\beta_{1} U^{*}+\beta_{2} I^{*}+\mu\right)+(\xi+\gamma)-\beta_{2} S^{*} e^{-\mu \tau} \\
= & \frac{(\mu+\sigma+\zeta) R_{0}\left(\beta_{1} \theta V+\beta_{2}(\xi+\gamma)\right)+\beta_{1} \theta V \mu\left(R_{0}-1\right) R_{0}}{R_{0}\left(\beta_{1} \theta V+\beta_{2}(\xi+\gamma)\right)} \\
& +\frac{\beta_{2} R_{0} \mu(\xi+\gamma) \mu\left(R_{0}-1\right)-\beta_{2} S_{0}\left(\beta_{1} \theta V+\beta_{2}(\xi+\gamma)\right)}{R_{0}\left(\beta_{1} \theta V+\beta_{2}(\xi+\gamma)\right)} \\
& +\frac{R_{0} \mu\left(\beta_{1} \theta V+\beta_{2}(\xi+\gamma)\right)+R_{0}(\xi+\gamma)\left(\beta_{1} \theta V+\beta_{2}(\xi+\gamma)\right)}{R_{0}\left(\beta_{1} \theta V+\beta_{2}(\xi+\gamma)\right)} \\
= & \frac{(\mu+\sigma+\zeta) R_{0} \beta_{1} \theta V+\beta_{1} \theta V \mu\left(R_{0}-1\right) R_{0}+\beta_{2} R_{0} \mu(\xi+\gamma) \mu\left(R_{0}-1\right)}{R_{0}\left(\beta_{1} \theta V+\beta_{2}(\xi+\gamma)\right)} \\
& +\frac{R_{0} \mu\left(\beta_{1} \theta V+\beta_{2}(\xi+\gamma)\right)+R_{0}(\xi+\gamma)\left(\beta_{1} \theta V+\beta_{2}(\xi+\gamma)\right)}{R_{0}\left(\beta_{1} \theta V+\beta_{2}(\xi+\gamma)\right)} \\
= & \frac{S_{0} \beta_{1} \theta V}{R_{0}(\xi+\gamma)}+\mu R_{0}+(\xi+\gamma)>0 .
\end{aligned}
$$

By computing we can obtain $\beta_{1} U^{*}+\beta_{2} I^{*}+\mu=\mu R_{0}$ and $R_{0}(\mu+\sigma+\zeta)(\xi+\gamma)=$ $\beta_{2} S_{0}(\xi+\gamma)+\theta V \beta_{1} S_{0}$, then we further have

$$
\begin{aligned}
p_{1}= & a_{1}-b_{1} \\
= & (\mu+\sigma+\zeta)(\xi+\gamma)+(\mu+\sigma+\zeta+\xi+\gamma)\left(\beta_{1} U^{*}+\beta_{2} I^{*}+\mu\right) \\
& -\beta_{2} S^{*}(\xi+\gamma) e^{-\mu \tau}-\theta V \beta_{1} S^{*} e^{-\mu \tau}-\mu \beta_{2} S^{*} e^{-\mu \tau} \\
= & \frac{1}{R_{0}}\left(R_{0}(\mu+\sigma+\zeta)(\xi+\gamma)+R_{0}^{2} \mu(\mu+\sigma+\zeta+\xi+\gamma)\right. \\
& \left.-\beta_{2} S_{0}(\xi+\gamma)-\theta V \beta_{1} S_{0}-\mu \beta_{2} S_{0}\right) \\
= & \beta_{2} \Lambda\left(\frac{R_{0}-1}{R_{0}}\right)+\frac{\beta_{1} \theta V \Lambda}{\xi+\gamma}+\frac{\beta_{2} \Lambda(\xi+\gamma)+\beta_{1} \theta V \Lambda}{\mu+\sigma+\zeta}>0, \\
p_{0}= & a_{0}-b_{0} \\
= & (\mu+\sigma+\zeta)\left(\beta_{1} U^{*}+\beta_{2} I^{*}+\mu\right)(\xi+\gamma)-(\xi+\gamma) \mu e^{-\mu \tau} \beta_{2} S^{*}-\theta V \mu \beta_{1} S^{*} e^{-\mu \tau} \\
= & (\mu+\sigma+\zeta)(\xi+\gamma) \mu R_{0}-\frac{1}{R_{0}}\left(\mu\left((\xi+\gamma) \beta_{2} S_{0}+\theta V \beta_{1} S_{0}\right)\right) \\
= & (\mu+\sigma+\zeta)(\xi+\gamma) \mu\left(R_{0}-1\right)>0 .
\end{aligned}
$$

Furthermore, by calculating it follows that

$$
\begin{aligned}
p_{1} p_{2}-p_{0}= & \left(\frac{S_{0} \beta_{1} \theta V}{R_{0}(\xi+\gamma)}+\mu R_{0}+(\xi+\gamma)\right)\left(R_{0} \mu(\mu+\sigma+\zeta)+R_{0} \mu(\xi+\gamma)\right. \\
& \left.-\frac{\mu \beta_{2} S_{0}}{R_{0}}\right)-(\mu+\sigma+\zeta)(\xi+\gamma) \mu R_{0}+(\mu+\sigma+\zeta)(\xi+\gamma) \mu>0 .
\end{aligned}
$$

Therefore, by the Hurwitz criterion equation (30) only has negative real roots. 
Let $\tau>0$. Assume that $\lambda=\omega i(\omega>0)$ is a pure imaginary root of equation (29). Substitute it into equation (29) and separate the imaginary and real parts by using $e^{-\lambda \tau}=\cos (\omega \tau)-i \sin (\omega \tau)$, we can get

$$
\left\{\begin{array}{l}
-\omega^{3}+a_{1} \omega=b_{2} \omega^{2} \sin (\omega \tau)+b_{1} \omega \cos (\omega \tau)-b_{0} \sin (\omega \tau) \\
-a_{2} \omega^{2}+a_{0}=-b_{2} \omega^{2} \cos (\omega \tau)+b_{1} \omega \sin (\omega \tau)+b_{0} \cos (\omega \tau)
\end{array}\right.
$$

Square and add the two equations in (31) we have

$$
\omega^{6}+p \omega^{4}+q \omega^{2}+r=0
$$

where $p=a_{2}^{2}-2 a_{1}-b_{2}^{2}, q=a_{1}^{2}-2 a_{0} a_{2}-b_{1}^{2}+2 b_{0} b_{2}$ and $r=a_{0}^{2}-b_{0}^{2}$. By directly calculating we can obtain that

$$
\begin{aligned}
p= & {\left[(\mu+\sigma+\zeta)+\left(\beta_{1} U^{*}+\beta_{2} I^{*}+\mu\right)+(\xi+\gamma)\right]^{2}-2[(\mu+\sigma+\zeta)(\xi+\gamma)} \\
& \left.+(\mu+\sigma+\zeta+\xi+\gamma)\left(\beta_{1} U^{*}+\beta_{2} I^{*}+\mu\right)\right]-\beta_{2}^{2} S^{* 2} e^{-2 \mu \tau} \\
= & (\mu+\sigma+\zeta)^{2}+\left(\beta_{1} U^{*}+\beta_{2} I^{*}+\mu\right)^{2}+(\xi+\gamma)^{2}-\beta_{2}^{2} S^{* 2} e^{-2 \mu \tau} \\
= & \frac{R_{0}^{2}\left(\beta_{1} \theta V+\beta_{2}(\xi+\gamma)\right)^{2}(\mu+\sigma+\zeta)^{2}+\beta_{1}^{2} \theta^{2} V^{2} \mu^{2}\left(R_{0}-1\right)^{2} R_{0}^{2}}{R_{0}^{2}\left(\beta_{1} \theta V+\beta_{2}(\xi+\gamma)\right)^{2}} \\
& +\frac{\beta_{2}^{2} R_{0}^{2}(\xi+\gamma)^{2} \mu^{2}\left(R_{0}-1\right)^{2}-\beta_{2}^{2} S_{0}^{2}\left(\beta_{1} \theta V+\beta_{2}(\xi+\gamma)\right)^{2} e^{-2 \mu \tau}}{R_{0}^{2}\left(\beta_{1} \theta V+\beta_{2}(\xi+\gamma)^{2}\right.} \\
& +\mu^{2}+2 \beta_{1} U^{*} \beta_{2} I^{*}+2 \beta_{1} U^{*} \mu+2 \beta_{2} I^{*} \mu(\xi+\gamma)^{2} \\
= & \frac{R_{0}^{2} \beta_{1}^{2} \theta^{2} V^{2}(\mu+\sigma+\zeta)^{2}+2 \beta_{1} \theta V \beta_{2}(\xi+\gamma)(\mu+\sigma+\zeta)^{2}}{R_{0}^{2}\left(\beta_{1} \theta V+\beta_{2}(\xi+\gamma)\right)^{2}} \\
& +\frac{\beta_{1}^{2} \theta^{2} V^{2} \mu^{2}\left(R_{0}-1\right)^{2} R_{0}^{2}+\beta_{2}^{2} R_{0}^{2}(\xi+\gamma)^{2} \mu^{2}\left(R_{0}-1\right)^{2}}{R_{0}^{2}\left(\beta_{1} \theta V+\beta_{2}(\xi+\gamma)\right)^{2}} \\
& +\mu^{2}+2 \beta_{1} U^{*} \beta_{2} I^{*}+2 \beta_{1} U^{*} \mu+2 \beta_{2} I^{*} \mu(\xi+\gamma)^{2}>0,
\end{aligned}
$$


and

$$
\begin{aligned}
& q=\left[(\mu+\sigma+\zeta)(\xi+\gamma)+(\mu+\sigma+\zeta)\left(\beta_{1} U^{*}+\beta_{2} I^{*}+\mu\right)+(\xi+\gamma)\left(\beta_{1} U^{*}+\beta_{2} I^{*}\right.\right. \\
& +\mu)]^{2}-2\left[(\mu+\sigma+\zeta)+\left(\beta_{1} U^{*}+\beta_{2} I^{*}+\mu\right)+(\xi+\gamma)\right]\left[( \mu + \sigma + \zeta ) \left(\beta_{1} U^{*}\right.\right. \\
& \left.\left.+\beta_{2} I^{*}+\mu\right)(\xi+\gamma)\right]-\left[\beta_{2} S^{*}(\xi+\gamma) e^{-\mu \tau}+\theta V \beta_{1} S^{*} e^{-\mu \tau}+\mu \beta_{2} S^{*} e^{-\mu \tau}\right]^{2} \\
& +2\left((\xi+\gamma) \mu e^{-\mu \tau} \beta_{2} S^{*}+\theta V \mu \beta_{1} S^{*} e^{-\mu \tau}\right) \beta_{2} S^{*} e^{-\mu \tau} \\
& =(\mu+\sigma+\zeta)^{2}(\xi+\gamma)^{2}+(\mu+\sigma+\zeta)^{2}\left(\beta_{1} U^{*}+\beta_{2} I^{*}+\mu\right)^{2} \\
& +(\xi+\gamma)^{2}\left(\beta_{1} U^{*}+\beta_{2} I^{*}+\mu\right)^{2}-\frac{1}{R_{0}^{2}}\left(\beta_{2}^{2} S_{0}^{2}(\xi+\gamma)^{2} e^{-2 \mu \tau}+\theta^{2} V^{2} \beta_{1}^{2} S_{0}^{2} e^{-2 \mu \tau}\right) \\
& -\frac{1}{R_{0}^{2}}\left(2 \beta_{2}^{2} S_{0}^{2} \mu(\xi+\gamma) e^{-2 \mu \tau}+\mu^{2} \beta_{2}^{2} S_{0}^{2} e^{-2 \mu \tau}+2 \beta_{2} \beta_{1} S_{0}^{2}(\xi+\gamma) \theta V e^{-2 \mu \tau}\right) \\
& +\frac{1}{R_{0}^{2}}\left(-2 \beta_{2} \beta_{1} S_{0}^{2} \theta V e^{-2 \mu \tau}+2 \beta_{2}^{2} S_{0}^{2} \mu(\xi+\gamma) e^{-2 \mu \tau}+2 \beta_{2} \beta_{1} S_{0}^{2} \theta V e^{-2 \mu \tau}\right) \\
& =\frac{1}{R_{0}^{2}}\left(R_{0}^{2}(\mu+\sigma+\zeta)^{2}(\xi+\gamma)^{2}+\mu^{2} R_{0}^{4}\left((\mu+\sigma+\zeta)^{2}+(\xi+\gamma)^{2}\right)\right) \\
& +\frac{1}{R_{0}^{2}}\left(-\beta_{2}^{2} S_{0}^{2}(\xi+\gamma)^{2} e^{-2 \mu \tau}-\theta^{2} V^{2} \beta_{1}^{2} S_{0}^{2} e^{-2 \mu \tau}-\mu^{2} \beta_{2}^{2} S_{0}^{2} e^{-2 \mu \tau}\right) \\
& +\frac{1}{R_{0}^{2}}\left(-2 \beta_{2} \beta_{1} S_{0}^{2}(\xi+\gamma) \theta V e^{-2 \mu \tau}-2 \beta_{2}^{2} S_{0}^{2} \mu(\xi+\gamma) e^{-2 \mu \tau}\right. \\
& \left.-2 \beta_{2} \beta_{1} S_{0}^{2} \theta V e^{-2 \mu \tau}\right)+\frac{1}{R_{0}^{2}}\left(2 \beta_{2}^{2} S_{0}^{2} \mu(\xi+\gamma) e^{-2 \mu \tau}+2 \beta_{2} \beta_{1} S_{0}^{2} \theta V e^{-2 \mu \tau}\right) \\
& =\beta_{2}^{2} \Lambda^{2} e^{-2 \mu \tau}+\frac{2 \beta_{2} \Lambda \beta_{1} \theta V \Lambda e^{-2 \mu \tau}}{\xi+\gamma}+\frac{\left(\beta_{2} \Lambda(\xi+\gamma) e^{-\mu \tau}+\beta_{1} \theta V \Lambda e^{\mu \tau}\right)^{2}}{(\mu+\sigma+\zeta)^{2}} \\
& +\frac{\beta_{1}^{2} \theta^{2} V^{2} \Lambda^{2} e^{-2 \mu \tau}}{(\xi+\gamma)^{2}}+\frac{1}{R_{0}^{2}}\left(\Lambda^{2} \beta_{2}^{2} e^{-2 \mu \tau}+2 \beta_{2} \beta_{1} S_{0}^{2}(\xi+\gamma) \theta V e^{-2 \mu \tau}\right. \\
& \left.-2 \beta_{2} \beta_{1} S_{0}^{2}(\xi+\gamma) \theta V e^{-2 \mu \tau}\right) \\
& =\beta_{2}^{2} \Lambda^{2} e^{-2 \mu \tau}\left(\frac{R_{0}^{2}-1}{R_{0}^{2}}\right)+\frac{\beta_{1}^{2} \theta^{2} V^{2} \Lambda^{2} e^{-2 \mu \tau}}{(\xi+\gamma)^{2}}+\frac{2 \beta_{2} \Lambda \beta_{1} \theta V \Lambda e^{-2 \mu \tau}}{\xi+\gamma} \\
& +\frac{\left(\beta_{2} \Lambda(\xi+\gamma) e^{-\mu \tau}+\beta_{1} \theta V \Lambda e^{\mu \tau}\right)^{2}}{(\mu+\sigma+\zeta)^{2}}>0 .
\end{aligned}
$$

Finally, we have

$$
r=a_{0}^{2}-b_{0}^{2}=\left(a_{0}-b_{0}\right)\left(a_{0}+b_{0}\right)=(\mu+\sigma+\zeta)^{2}(\xi+\gamma)^{2} \mu^{2}\left(R_{0}^{2}-1\right)>0 .
$$

This shows that equation (32) has not positive root. Therefore, the characteristic equation (29) does not have pure imaginary roots when $\tau>0$. This further implies that the equation (29) does not have a root with a positive real part for any $\tau \geq 0$. Therefore, the endemic equilibrium $E^{*}$ is locally asymptotically stable for any $\tau \geq 0$.

Remark 6 In Theorems 7 and 8 only the local asymptotic stability of disease-free equilibrium $E_{0}$ and unique endemic equilibrium $E^{*}$ is established. Whether we can further 
construct the appropriate Lyapunov function and further use the LaSalle invariant principle or use the geometric method to get the global stability of $E_{0}$ and $E^{*}$, which is still an open problem.

\subsection{Uniform persistence}

In this part, we investigate the uniform persistence of model (20). Here, we call that model (20) is uniformly persistent if there is a constant $\varepsilon>0$ such that for any positive solution $(S(t), I(t), U(t))$ of model (20) one has $\liminf _{t \rightarrow \infty} S(t) \geq \varepsilon$, $\liminf \operatorname{in}_{t \rightarrow \infty} I(t) \geq \varepsilon$ and $\liminf _{t \rightarrow \infty} U(t) \geq \varepsilon$. However, it is with regret that we here only can establish the uniform persistence criterion for the special case $\beta_{2}=0$ in model (20). That is, in model (20) there is not the direct transmission of the disease by the infected individual.

Denote by $(S(t, v), I(t, v), U(t, v))$ the solution of model (20) with initial function $v=\left(v_{1}, v_{2}, v_{3}\right) \in C\left([-\tau, 0], R_{+}^{3}\right)$ at time $t=0$. Define the $\left(S_{t}(v), I_{t}(v), U_{t}(v)\right)=$ $(S(t+\theta, v), I(t+\theta, v), U(t+\theta, v))$ for any $t>0$ and $\theta \in[-\tau, 0]$. Further define the set

$$
X=\left\{\left(v_{1}, v_{2}, v_{3}\right) \in C\left([-\tau, 0], R_{+}^{3}\right): v_{2} \neq 0, v_{3} \neq 0\right\}
$$

The boundary of $X$ is

$$
\partial X:=C\left([-\tau, 0], R_{+}^{3}\right) / X=\left\{\left(v_{1}, v_{2}, v_{3}\right) \in C\left([-\tau, 0], R_{+}^{3}\right): v_{2} \equiv 0 \text { or } v_{3} \equiv 0\right\} .
$$

We have theorem as given below.

Theorem 9 Assume $R_{0}>1$ and $\beta_{2}=0$. Then there is a constant $\varepsilon>0$, so that any positive solution $S(t, v), I(t, v), U(t, v)$ of model (20) with initial function $v=$ $\left(v_{1}, v_{2}, v_{3}\right) \in X$ one has

$$
\liminf _{t \rightarrow \infty} S(t, v) \geq \varepsilon, \liminf _{t \rightarrow \infty} I(t, v) \geq \varepsilon, \liminf _{t \rightarrow \infty} U(t, v) \geq \varepsilon
$$

Proof Firstly, when $\beta_{2}=0$ we have $R_{0}=\frac{\beta_{1} \theta V S_{0} e^{-\mu \tau}}{(\mu+\sigma+\zeta)(\xi+\gamma)}$. Let $u(t, v)=$ $(S(t, v), I(t, v), U(t, v))$ be the solution of model (20) with initial conditions (19), then from the first equation of model (20) we know that

$$
\left.\frac{d S(t, v)}{d t}=\Lambda-\left(\mu+\beta_{1} U(t, v)\right)\right) S(t, v) \geq \Lambda-\left(\mu+\beta_{1}\right) S(t, v) .
$$

Using the comparison principle we easily know that $\lim _{t \rightarrow+\infty} \inf S(t, v) \geq \frac{\Lambda}{\mu+\beta_{1}}$. This shows that variable $S(t)$ in model (20) is uniformly persistent.

we can clear see that $X$ is the invariant set for model (20). Furthermore, define

$$
M_{\partial}=\left\{v \in C\left([-\tau, 0], R_{+}^{3}\right):\left(S_{t}(v), I_{t}(v), U_{t}(v)\right) \in \partial X, \forall t \geq 0\right\} .
$$


Let $M_{0}=\left\{E_{0}\right\}$. Clearly, we have $M_{0} \subset \cup_{v_{0} \in M_{\partial}} \omega\left(v_{0}\right)$, where $\omega\left(v_{0}\right)$ indicates the $\omega$ limit set of solution $\left(S_{t}\left(v_{0}\right), I_{t}\left(v_{0}\right), U_{t}\left(v_{0}\right)\right)$ with initial value $u(0)=v_{0}$. For any given $v \in M_{\partial}$, since $\left(S_{t}(v), I_{t}(v), U_{t}(v)\right) \in M_{\partial}$ for all $t \geq 0$, clearly we have $I(t, v) \equiv 0$ or $U(t, v) \equiv 0$.

If $I(t, v) \equiv 0$, since $S(t, v)$ has a positive lower boundary, then from the second equation of model (20) we can obtain $U(t, v) \equiv 0$. Thus, from model (20) is reduced to the following subsystem as follows

$$
\frac{d S(t, v)}{d t}=A-\mu S(t, v)
$$

From this, we can get $\lim _{t \rightarrow \infty} S(t, v)=S_{0}$ which implies $\omega\left(v_{0}\right)=\left\{E_{0}\right\}$.

If $U(t, v) \equiv 0$, then from the third equation of model (20) we easily can get $I(t, v) \equiv 0$. Similarly, we also have subsystem (33). Thus, $\lim _{t \rightarrow \infty} S(t, v)=S_{0}$ which also implies $\omega\left(v_{0}\right)=\left\{E_{0}\right\}$. From the above discussion we can get $\cup_{v_{0} \in M_{\partial}} \omega\left(v_{0}\right) \subset M_{0}$. Thence, finally we have $M_{0}=\cup_{v_{0} \in M_{\partial}} \omega\left(v_{0}\right)$.

Now, we are going to prove that $K^{s}\left(E_{0}\right) \cap X=\emptyset$, where $K^{s}\left(E_{0}\right)$ is the stable set of $E_{0}$. Assuming to have a $v \in X$ such that $\lim _{t \rightarrow \infty} u(t, v)=E_{0}$. Since $R_{0}>1$, there is an enough small $\varepsilon>0$ so that

$$
\frac{\beta_{1}\left(S_{0}-\varepsilon\right) \theta \frac{\omega}{\alpha} e^{-\mu \tau}}{(\mu+\sigma+\zeta)(\xi+\gamma)}-1>0
$$

Furthermore, for this $\varepsilon>0$, there is a $t^{*}>0$ so that $S(t, v) \geq S_{0}-\varepsilon, I(t, v)<\varepsilon$ and $U(t, v)<\varepsilon$ for all $t \geq t^{*}$. Define the function as given below

$$
L(t, v)=e^{\mu \tau} I(t, v)+\frac{\mu+\sigma+\zeta}{\theta \frac{\omega}{\alpha} e^{-\mu \tau}} U(t, v)+\int_{t-\tau}^{t} \beta_{1} U(x) S(x) d x
$$

Then $\lim _{t \rightarrow \infty} L(t, v)=0$. When $t \geq t^{*}$ we have

$$
\begin{aligned}
\frac{d L(t, v)}{d t}= & e^{\mu \tau} \beta_{1} U(t-\tau, v) S(t-\tau, v) e^{-\mu \tau}-e^{\mu \tau} I(t, v)(\mu+\sigma+\zeta) \\
& +\beta_{1} U(t, v) S(t, v)-\beta_{1} U(t-\tau, v) S(t-\tau, v)+e^{\mu \tau} I(t, v)(\mu+\sigma+\zeta) \\
& -\frac{(\mu+\sigma+\zeta)(\xi+\gamma) U(t, v)}{\theta \frac{\omega}{\alpha} e^{-\mu \tau}} \\
= & \beta_{1} U(t, v) S(t, v)-\frac{(\mu+\sigma+\zeta)(\xi+\gamma) U(t, v)}{\theta \frac{\omega}{\alpha} e^{-\mu \tau}} \\
\geq & {\left[\frac{\beta_{1}\left(S_{0}-\varepsilon\right) \theta \frac{\omega}{\alpha} e^{-\mu \tau}}{(\mu+\sigma+\zeta)(\xi+\gamma)}-1\right] \frac{(\mu+\sigma+\zeta)(\xi+\gamma)}{\theta \frac{\omega}{\alpha} e^{-\mu \tau}} U(t, v) . }
\end{aligned}
$$

Clearly, we have $\frac{d L(t, v)}{d t}>0$ for all $t \geq t^{*}$. Therefore, $L(t, v)$ is an increasing function on $t \geq t^{*}$. It can be seen that $\lim _{t \rightarrow \infty} L(t, v) \neq 0$, which leads to a contradiction. Hence, we have $K^{s}\left(E_{0}\right) \cap X=\emptyset$. Depending on the persistence theory of dynamical systems, we clearly see that the existence of constant $\varepsilon$ makes the inequality given 
below hold for any $v_{0} \in X$.

$$
\liminf _{t \rightarrow \infty} S(t, v) \geq \varepsilon, \liminf _{t \rightarrow \infty} I(t, v) \geq \varepsilon, \liminf _{t \rightarrow \infty} U(t, v) \geq \varepsilon
$$

Finally, we come to conclusion that the positive solutions for model (20) is uniformly persistent. This completes the proof.

Remark 7 In Theorem 8 we have obtained that when $R_{0}>1$ the unique disease-free equilibrium $E_{0}$ is unstable for model (20). Since the positive solutions of model (20) also are ultimately bounded, so long as we can prove $K^{s}\left(E_{0}\right) \cap X=\emptyset$, then from the persistence theory of dynamical systems we exactly come to conclusion that the positive solutions for model (20) is uniformly persistent. But, unfortunately when $\beta_{2}>0$ we now can not obtain $K^{s}\left(E_{0}\right) \cap X=\emptyset$. Therefore, an interesting open problem is when $\beta_{2}>0$ to prove $K^{s}\left(E_{0}\right) \cap X=\emptyset$.

Remark 8 We can propose a more general open problem. That is, only when $R_{0}>1$, or when $R_{0}>1$ and some additional condition is added, to prove that the general model (20), especially $\beta_{2}>0$ is requested, is uniformly persistent.

Remark 9 From Theorem 8 and the explanation of basic reproduction numbers $R_{0}^{(1)}$ and $R_{0}^{(2)}$, we see that increasing the production rate $\alpha$ of $B$ cells, the clearance rate $\gamma$ of environmental virus and the quarantine and cure rate $\sigma$ of infected individuals, and lengthening the incubation period $\tau$ can effectively retard the outbreak of disease transmission.

\section{Numerical examples}

In this section, we verified the open problems on the stability of antibody response infection equilibrium for model (3), the global stability of $E_{0}$ and $E^{*}$ for model (2) and the uniform persistence of positive solutions for general model (20) proposed in Remarks 1, 6 and 8 by means of the numerical examples.

Example 1 In model (3), we take the parameters $\Lambda_{c}=60, w=400, k=$ $1.5 \times 10^{-4}, m=0.02, d=0.02, p=10, c=15, \tau_{1}=0.2, \alpha=$ $0.0009999, q=0.03, \omega=0.02$ and $U=0.5$. Besides, the initial values of solutions $\left(T(t), T^{*}(t), V(t), B(t)\right)$ are respectively given as $(1800,1000,50,1700)$, $(1300,800,100,1400),(750,250,75,1700)$ and $(1340,500,35,1330)$.

By calculation we have $R_{w}=7.1622>1$. Then from the numerical simulations in Fig. 2 we see that the antibody response infection equilibrium $A_{2}(U)=$ (2608.7, 194.8881, 20.0020, 3081.1) of model (3) may be globally asymptotically stable. Thence, the theoretical conclusion in Theorem 4 is verified, and the open problem proposed in Remark 1 would be reasonable.

Example 2 In models (2) and (3), we choose the parameters $\Lambda=50, \beta_{1}=0.05$, $\beta_{2}=0.005, w=4 \times 10^{5}, \mu=0.44, \gamma=0.151, \theta=1.4 \times 10^{-6}, \Lambda_{c}=9000$, 

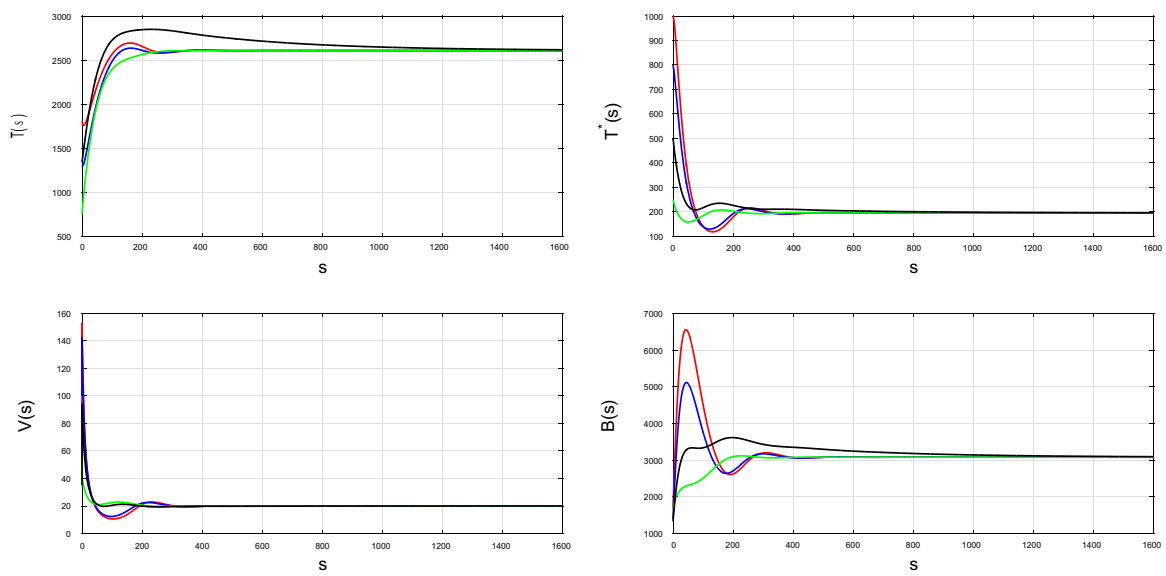

Fig. 2 Dynamical behaviors of $T(s), T^{*}(s), V(s), B(s)$. From the numerical simulation we know that the solution converge to the antibody response infection equilibrium $A_{2}(U)=$ $(2608.7,194.8881,20.0020,3081.1)$
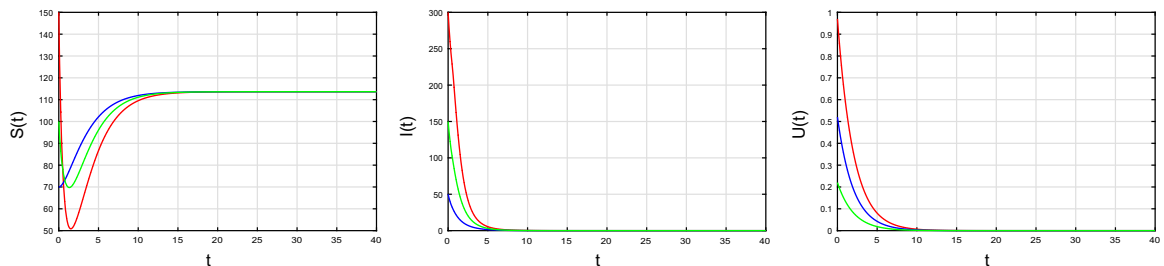

Fig. 3 Dynamical behaviors of $S(t), I(t), U(t)$. From the numerical simulation we know that the solution converge to disease-free equilibrium $E_{0}=(113.6868,0,0)$

$k=1.05 \times 10^{-6}, m=0.07, d=0.07, p=95, c=61, \tau_{1}=0.5, \tau=0.8$, $\sigma=0.5, \alpha=0.04, \zeta=0.32, \xi=0.35$ and $\omega=0.5$. Besides, the initial values of solutions $(S(t), I(t), U(t))$ are respectively given as $(150,300,0.97),(70,50,0.52)$ and $(100,150,0.22)$.

By calculation we have $R_{w 1}=1.4498>1$, and hence, $R_{w}>1$ for any $U>0$. We also have $R_{0}=0.3620<1$. Then from the numerical simulations in Fig. 3 we see that disease-free equilibrium $E_{0}=(113.6868,0,0)$ of model (2) is globally asymptotically stable. Thus, the theoretical conclusion in Theorem 7 is verified, and the open problem proposed in Remark 6 would be reasonable.

Example 3 In models (2) and (3), we choose the parameters $\Lambda=50, \beta_{1}=0.05$, $\beta_{2}=0.06, w=4 \times 10^{5}, \mu=0.64, \gamma=0.151, \theta=1.4 \times 10^{-6}, \Lambda_{c}=6000$, $k=1.05 \times 10^{-6}, m=0.07, d=0.07, p=95, c=50, \tau_{1}=0.5, \tau=0.5, \sigma=0.05$, $\alpha=0.0004, \zeta=0.0012, \xi=0.0095$ and $\omega=0.9$. Besides, the initial values of solutions $(S(t), I(t), U(t))$ are respectively given as $(100,100,0.97),(70,50,0.52)$ and $(100,75,0.22)$.

By calculation we have $R_{w 1}=1.1409>1$ and hence, $R_{w}>1$ for any $U>0$. We also have $R_{0}=5.0051>1$. Then from the numerical simulations in Fig. 4 we 

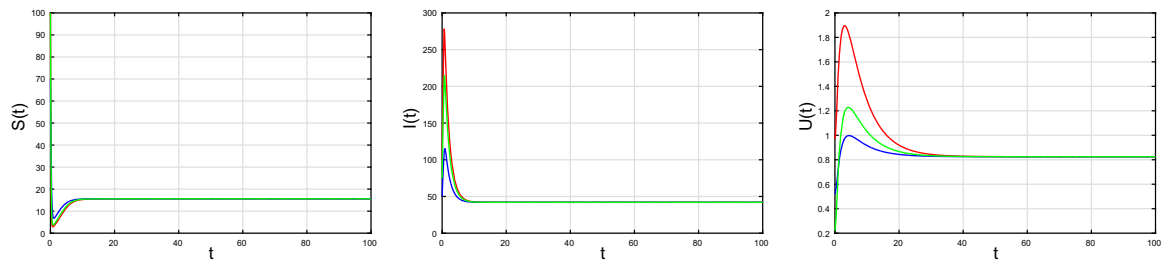

Fig. 4 Dynamical behaviors of $S(t), I(t), U(t)$. From the numerical simulation we know that the solution converge to disease-free equilibrium $E^{*}=(15.6092,42.0331,0.8249)$

see that the endemic equilibrium $E^{*}=(15.6092,42.0331,0.8249)$ of model (2) may be globallly asymptotically stable. Thus, the theoretical conclusion in Theorem 8 is verified, and the open problem proposed in Remark 8 can be reasonable.

\section{Conclusion}

In this work, we mainly focus on the dynamics in a disease transmission model coupled virus infection in host with incubation delay and environmental effects. The model is divided into two different time scales dynamics.

For the virus infection model in host with antibody immune, latent delay and environmental virus invading, the main results on the existence of antibody-free and antibody response infection equilibria and the threshold criteria of local and global stability for equilibria are established. The research results show that the environmental virus invading leads to that the virus infection in host always occurs. The short latent period can retard the outbreak of virus infection in host and increasing the production rate of $\mathrm{B}$ cells will decrease the number of virus and then retard the virus infection in host.

For the disease transmission model with incubation delay and immune response in host, the basic reproduction number $R_{0}$ is defined, and the stability of equilibria are established. When $R_{0} \leq 1$ the disease-free equilibrium is locally asymptotically stable, and when $R_{0}>1$ the model has a unique endemic equilibrium which is locally asymptotically stable, and the disease prevalence is uniform persistent. Based on these conclusions and the explanation of basic reproduction numbers $R_{0}^{(1)}$ and $R_{0}^{(2)}$, we see that increasing the production rate of B cells, the clearance rate of environmental virus and the cure rate of infected individuals, and shortened the incubation period can effectively retard the outbreak of disease transmission.

Some open problems are also presented in this paper. For the virus infection model in host, the open problem on the global stability of antibody response infection equilibrium is proposed in Remark 1. From Example 1 presented in Sect. 5, we can conjecture that this equilibrium is globally asymptotically stable. For the disease transmission model, a series of open problems on the global stability of disease-free and endemic equilibria and the uniform persistence of positive solutions for general model (20) are proposed in Remarks 6 and 8, respectively. From Examples 2 and 3 presented in Sect. 5 , we can conjecture that when basic reproduction number $R_{0}<1$ then the disease- 
free equilibrium is globally asymptotically stable, and when $R_{0}>1$ then the model is uniform persistent and the endemic equilibrium is also globally asymptotically stable.

There are some new research subjects involving the dynamics in a disease transmission model coupled virus infection in host with environmental effects in the future. Firstly, in this paper we know that only bilinear incidence is considered. Therefore, more general models with nonlinear incidence also should be discussed. Next, in this paper we only discussed the case of antibody response in host. However, more complex situation is that there is no antibody response in host. In this case, according to assumption $\left(\mathbf{H}_{\mathbf{2}}\right)$, in the disease transmission model (2) we will have $V(s) \rightarrow V_{1}(U)=\frac{1}{c}\left(g(U)+p T_{1}^{*}(U)\right)$ as $s \rightarrow \infty$ (See Theorem 2 in Sect. 3), model (2) will change into a more complex form. Therefore, the research on the dynamical behavior will be a very challenging subject. Thirdly, we have known that in many epidemic diseases such as Hepatitis, Tuberculosis, AIDS and COVID-19, the infected individuals in incubation periods also can possess the infection ability. Therefore, It is more meaningful to study the disease transmission model coupled virus infection with the incubation period infection. Fourthly, in fourth equation of model (2), the amount of virus $V$ can be from other populations other than people, for example, bats for Ebola. Therefore, it will be very interesting to consider such factors in our model (2). Lastly, when a disease spreads within a community, individuals acquire knowledge about this disease, Therefore, it will be interesting to study the memory effect on the dynamics of infectious model by using the new generalized fractional derivative presented in [45].

Acknowledgements This work was supported by the National Natural Science Foundation of China [Grant Nos. 11771373, 11861065] and the Natural Science Foundation of Xinjiang Province of China [Grant Nos. 2019D01C076].

\section{Declarations}

Conflict of interest The authors declare that they have no conflict of interest.

\section{References}

1. Anguelov, R., Banasiak, J., Bright, C., Lubuma, J., Ouifki, R.: The big unknown: the asymptomatic spread of COVID-19. Biomath. Forum. 9, 2005103 (2020)

2. Naheed, A.: A study of spatio-temporal spread of infectious disease: SARS. Bull. Aust. Math. Soc. 94, 522-523 (2016)

3. Deyle, R.E., Maher, M.C., Hernandez, R.D., Basu, S., Sugihara, G.: From the cover: global environmental drivers of influenza. Proc. Natl. Acad. Sci. U. S. A. 113, 13081-13086 (2016)

4. Lawrence, P., Danet, N., Reynard, O., Volchkova, V., Volchkov, V.: Human transmission of Ebola viruses. Curr. Opin. Virol. 22, 51-58 (2017)

5. Tahir, M., Shah, S., Zaman, G., Khan, T.: Stability behaviour of mathematical model MERS corona viruses spread in population. Filomat 33, 3947-3960 (2019)

6. Rhoubari, Z.E., Hattaf, K., Yousfi, N.: A class of Ebola virus disease models with post-death transmission and environmental contamination. Math. Model. Anal. Infect. Dis. (2020)

7. Feng, Z.L., Velasco-Hernandez, J., Tapia-Santos, B., Leite, C.A.: A model for coupling within-host and between-host dynamics in an infectious disease. Nonlinear Dyn. 68, 401-411 (2012) 
8. Lu, J.J., Teng, Z.D., Li, Y.K.: An age-structured model for coupling within-host and between-host dynamics in environmentally-driven infectious diseases. Chaos Solit. Fract. 139, 110024 (2020)

9. Wang, N., Zhang, L., Teng, Z.D.: A reaction-diffusion model for nested within-host and between-host dynamics in an environmentally-driven infectious disease. J. Appl. Anal. Comput. 11, 1898-1926 (2021)

10. Feng, Z.L., Cen, X.L., Zhao, Y.L., Velasco-Hernandez, J.: Coupled within-host and between-host dynamics and evolution of virulence. Math. Biosci. 270, 204-212 (2015)

11. Feng, Z.L., Velasco-Hernandez, J., Tapia-Santos, B.: A mathematical model for coupling within-host and between-host dynamics in an environmentally infectious disease. Math. Biosci. 241, 49-55 (2013)

12. Cen, X.L., Feng, Z.L., Zhao, Y.L.: Emerging disease dynamics in a model coupling within-host and between-host systems. J. Theor. Biol. 361, 141-151 (2014)

13. Artstein, Z.: The limiting equations of nonautonomous ordinary differential equations. J. Differ. Equ. 25, 184-202 (1977)

14. O’Malley, R.E.: Introduction to Singular Perturbations. Academic Press, New York (1974)

15. Artstein, Z.: Singularly perturbed ordinary differential equations with nonautonomous fast dynamics. J. Dyn. Differ. Equ. 11, 297-318 (1999)

16. Kato, J., Martynyuk, A.A., Shestakov, A.A.: Stability of Motion of Nonautonomous Systems (Method of Limiting Equations). Gordon \& Breach Publishers Amsterdam (1996)

17. Wilson, H.K.: Gauge functions and limit sets for nonautonomous ordinary differential equations. Proc. Am. Math. Soci. 35, 487-490 (1972)

18. Kiguradze, I.T., Chanturiya, T.A.: Asymptotic properties of solutions of nonautonomous ordinary differential equations. Desalination 241, 218-226 (2009)

19. Wen, B.Y., Wang, J.P., Teng, Z.D.: A discrete-time analog for coupled within-host and between-host dynamics in environmentally driven infectious disease. Adv. Differ. Equat. 2018, 69 (2018)

20. Wen, B.Y., Teng, Z.D.: Dynamical behaviour in discrete coupled within-host and between-host epidemic model with environmentally driven and saturation incidence. J. Differ. Equ. Appl. 18, 1-22 (2021)

21. Gilchrist, M.A., Coombs, D.: Evolution of virulence: interdependence, constraints, and selection using netsed models. Theor. Popul. Biol. 69, 145-153 (2006)

22. Mideo, N., Alizon, S., Day, T.: Linking within-host and between-host dynamics in the evolutionary epidemiology of infectious diseases. Trends Ecol. Evol. 23, 511-517 (2008)

23. Gilchrist, M.A., Sasaki, A.: Modeling host-parasite coevolution: a nested approach based on mechanistic models. Theor. Biol. 218, 289-308 (2002)

24. Coombs, D., Gilchrist, M.A., Ball, C.L.: Evaluating the importance of within-and between-host selction pressures on the evolution of chronic pathogens. Theor. Popul. Biol. 72, 576-591 (2007)

25. National Health Commission of the People's Republic of China. (2020). http://www.nhc.gov.cn/. Accessed 26 Jan 2020

26. Jiao, J.J., Liu, Z.Z., Cai, S.H.: Dynamics of an SEIR model with infectivity in incubation period and homestead-isolation on the susceptible. Appl. Math. Lett. 107, 106442 (2020)

27. Hethcote, H.W., Driessche, P.: An SIS epidemic model with variable population size and a delay. J. Math. Biol. 34, 177-194 (1995)

28. Hethcote, H.W., Driessche, P.: Two SIS epidemilogic models with delays. J. Math. Biol. 40, 3-26 (2000)

29. Xu, R.: Global dynamics of an SEIS epidemiological model with time delay describing a latent period. Math. Comput. Simulat. 85, 90-102 (2012)

30. Cooke, K.L., Driessche, P.: Analysis of an SEIRS epidemic model with two delays. J. Math. Biol. 35, 240-260 (1996)

31. Kajiwara, T., Sasaki, T., Otani, Y.: Global stability for an age-structured multistrain virus dynamics model with humoral immunity. J. Appl. Math. Comput. 62, 239-279 (2020)

32. Feng, Z.L., Huang, W.Z., Castillo-Chavez, C.: On the role of variable latent periods in mathematical models for tuberculosis. J. Dyn. Differ. Equ. 13, 425-452 (2001)

33. Huo, H.F., Feng, L.X.: Global stability for an HIV/AIDS epidemic model with different latent stages and treatment. Appl. Math. Model. 37, 1480-1489 (2013)

34. Wang, S.F., Zou, D.Y.: Global stability of in-host viral models with humoral immunity and intracellular delays. Appl. Math. Model. 36, 1313-1322 (2012)

35. Elaiw, A.M.: Global stability analysis of humoral immunity viruses dynamics model including latently infected cells. J. Biol. Dynam. 9, 215-228 (2015) 
36. Gandolfi, A., Pugliese, A., Sinisgalli, C.: Epidemic dynamics and host immune response: a nested approach. J. Math. Biol. 70, 399-435 (2015)

37. Tang, S.T., Teng, Z.D., Miao, H.: Global dynamics of a reaction-diffusion viruses infection model with humoral immunity and nonlinear incidence. Comput. Math. Appl. 78, 786-806 (2019)

38. Xiang, H., Tang, Y.L., Huo, H.F.: A viral model with intracellular delay and humoral immunity. Bull. Malays. Math. Sci. Soc. 40, 1011-1023 (2017)

39. Guo, T., Qiu, Z.P., Rong, L.B.: Analysis of an HIV model with immune responses and cell-to-cell transmission. Bull. Malays. Math. Sci. Soc. 43, 581-607 (2020)

40. Hattaf, K.: Global stability and Hopf bifurcation of a generalized viral infection model with multidelays and humoral immunity. Phys. A 545, 123689 (2020)

41. Deans, J.A., Cohen, S.: Immunology of malaria. Ann. Rev. Microbiol. 37, 25-49 (1983)

42. Murase, A., Sasaki, T., Kajiwara, T.: Stability analysis of pathogen-immune interaction dynamics. J. Math. Biol. 51, 247-267 (2005)

43. Kuang, Y.: Delay Differential Equations with Applications in Population Dynamics. Academic Press, Boston (1993)

44. Driessche, P., Watmough, J.: Reproduction numbers and sub-threshold endemic equilibria for compartmental models of disease transmission. Math. Biosci. 180, 29-48 (2002)

45. Hattaf, K.: A new generalized definition of fractional derivative with non-singular kernel. Computation 49, 8020049 (2020)

Publisher's Note Springer Nature remains neutral with regard to jurisdictional claims in published maps and institutional affiliations. 